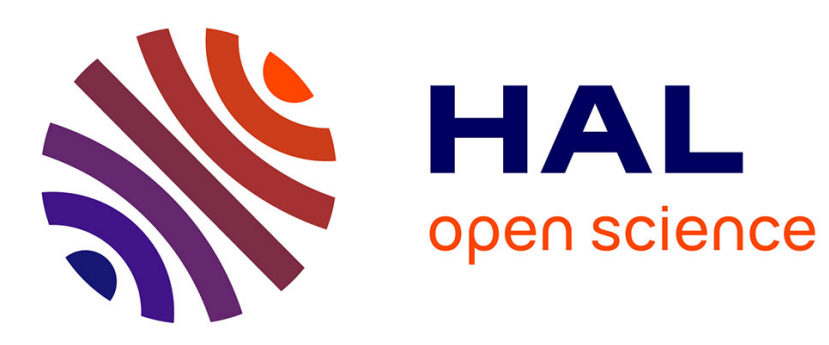

\title{
Stochastic analysis of spatial and opportunistic Aloha
}

François Baccelli, Bartlomiej Blaszczyszyn, Paul Mühlethaler

\section{To cite this version:}

François Baccelli, Bartlomiej Blaszczyszyn, Paul Mühlethaler. Stochastic analysis of spatial and opportunistic Aloha. IEEE Journal on Selected Areas in Communications, 2009, STOCHASTIC GEOMETRY AND RANDOM GRAPHS FOR THE ANALYSIS AND DESIGN OF WIRELESS NETWORKS, 27 (7), pp.1105 - 1119. 10.1109/JSAC.2009.090908 . inria-00360800v2

\section{HAL Id: inria-00360800 https://hal.inria.fr/inria-00360800v2}

Submitted on 4 May 2009

HAL is a multi-disciplinary open access archive for the deposit and dissemination of scientific research documents, whether they are published or not. The documents may come from teaching and research institutions in France or abroad, or from public or private research centers.
L'archive ouverte pluridisciplinaire HAL, est destinée au dépôt et à la diffusion de documents scientifiques de niveau recherche, publiés ou non, émanant des établissements d'enseignement et de recherche français ou étrangers, des laboratoires publics ou privés. 


\title{
Stochastic Analysis of Spatial and Opportunistic Aloha
}

\author{
François Baccelli*, Barłomiej Błaszczyszyn ${ }^{\dagger}$ and Paul Mühlethaler ${ }^{\ddagger}$
}

\begin{abstract}
Spatial Aloha is probably the simplest medium access protocol to be used in a large mobile ad hoc network: each station tosses a coin independently of everything else and accesses the channel if it gets heads. In a network where stations are randomly and homogeneously located in the Euclidean plane, there is a way to tune the bias of the coin so as to obtain the best possible compromise between spatial reuse and per transmitter throughput. This paper shows how to address this questions using stochastic geometry and more precisely Poisson shot noise field theory. The theory that is developed is fully computational and leads to new closed form expressions for various kinds of spatial averages (like e.g. outage, throughput or transport). It also allows one to derive general scaling laws that hold for general fading assumptions. We exemplify its flexibility by analyzing a natural variant of Spatial Aloha that we call Opportunistic Aloha and that consists in replacing the coin tossing by an evaluation of the quality of the channel of each station to its receiver and a selection of the stations with good channels (e.g. fading) conditions. We show how to adapt the general machinery to this variant and how to optimize and implement it. We show that when properly tuned, Opportunistic Aloha very significantly outperforms Spatial Aloha, with e.g. a mean throughput per unit area twice higher for Rayleigh fading scenarios with typical parameters.
\end{abstract}

Index Terms-MAC layer, multiple access, outage, throughput, transport capacity, network design, optimization, point process, signal to interference ratio, stochastic geometry, stochastic process.

\section{INTRODUCTION}

This paper contains several advances on the mathematical analysis of Spatial Aloha in the context of large Mobile Ad hoc Network (MANETs) as well as a discussion of a new variant of the protocol that we call Opportunistic Aloha.

\footnotetext{
*INRIA/ENS, 45 rue d'Ulm, 75005 Paris FRANCE, Francois.Baccelli@ens.fr

† INRIA/ENS and Math. Inst., Univ. of Wrocław, 45 rue d’Ulm, 75005 Paris FRANCE, Bartek.Blaszczyszyn@ens.fr

†INRIA, Le Chesnay, FRANCE, Paul.Muhlethaler@inria.fr
}

The paper builds upon the methodology proposed in [1], where stochastic geometry was first used for the quantitative analysis of this MAC protocol within this context. In MANETs the wireless nodes are randomly displayed and stochastic geometry can be used to provide closed form expressions for various kinds of spatial averages pertaining to the performance analysis of this MAC protocol. These spatial averages can be seen as empirical averages made over a large network and are particularly meaningful within this MANET context.

The advances of the present paper are of four different types:

1) Advances on stochastic modeling: in $\S$ II, we revisit the setting of [1] and we clarify and extend the fading scenarios for which the proposed analysis can be used. The connections between the Rayleigh model and the exponential assumptions of [1] are deepened and the justifications of certain independence assumptions are clarified. In addition, a more general framework is also introduced allowing one to analyze other types of fading (e.g. Rice or Nakagami) or to approximate the case with no fading at all.

2) Advances on performance metrics: we define a variety of new spatial performance metrics that we believe to be natural within this MANET context. In $\S$ III we go beyond the probability of success/outage and the mean number of successful transmissions per unit area, which were already considered in [1], to also consider the mean throughput (bit-rate) of a typical transmission, the mean throughput per unit area, the mean number of meters of progress per unit area, as well as the more general notion of density of transport of the protocol. The notion of spatial reuse as defined in VI is also shown to be quite useful to compare scenarios and policies. These definitions are of general interest and can be extended to other MAC protocols.

3) Advances on the mathematical evaluation of spatial averages: several new results on Spatial Aloha are derived. In [1], the main focus was 
on the evaluation of the probability of outage and on the associated optimization of the MAC parameters that are appropriate for large MANETs; the main new analytical result of the present paper are closed form expressions based on Fourier transform analysis; this bears for instance on spatial averages of the throughput ( $\S$ II-D) or the outage for general fading ( $\S$ II-C) and on the optimizations associated with these metrics $(\S \mathrm{V})$.

4) Advances on MAC protocol synthesis: in $\S$ VII, we illustrate the use the machinery developed for Spatial Aloha to synthesize a new variant of the protocol, that we call Opportunistic Aloha. HSDPA improves on plain CDMA by assessing the channel characteristics and scheduling transmissions in an opportunistic way that leverages the knowledge of the channel conditions. In the same vein, Opportunistic Aloha improves on Spatial Aloha by selecting transmitters having good channel conditions to their receivers. We show how to optimally tune the parameters of this MAC protocol and we evaluate the performance improvement that results from this adaptive variant of Spatial Aloha. The implementability of this protocol is discussed in $\S$ VIII based on our previous analysis and on discrete event simulation.

The general setting, which is described in detail in $\S$ II, is similar to that considered in [1]. It consists of a large MANET where all nodes have an infinite backlog of packets to transmit. The MAC is a slotted version of Aloha: at each time slot, each node independently tosses a coin with some bias $p$ which will be referred to as the medium access probability (MAP); it accesses the medium if the outcome is heads and it delays its transmission otherwise. The time-slot synchronization can be obtained through GPS or using atomic clocks in the network nodes. The nodes can also use common clocks with a distributed synchronization protocol. The quality of each wireless link is determined by the Signal to Interference and Noise Ratio (SINR) at the receiver. In the outage scenario, a packet transmission succeeds if the SINR at the receiver is larger than some predefined threshold. In the adaptive rate scenario, one is interested in the throughput obtained by each authorized transmission, which is also determined by the SINR at the receiver through some Shannon-like formula.

One of the general aims within the context of a large MANET is to tune the value of the MAP $p$ so as to obtain a good "social compromise" between the two contradicting aims of having, on the one hand a large average number of concurrent transmissions per unit area and on the other hand, a high probability that an authorized transmission will be successful / have high throughput: large values of $p$ allow more concurrent transmissions and consequently statistically larger interference, making the SINR smaller and transmissions more vulnerable; smaller values of $p$ give fewer transmissions with higher probability of success / throughput.

We assume that each transmitter has its one hop receiver at some fixed distance $r$. As we will see, another important tradeoff is associated with this onehop distance of transmissions $r$. A small $r$ makes the transmissions more sure/efficient but involves more relaying nodes to communicate packets between origin and destination. On the other hand, a larger one-hop distance reduces the number of hops but might increase the number of failed / small throughput transmissions at each hop.

Let us conclude this introduction by reviewing the relationships between the present paper and the literature on the matter.

In [2], Ehsan and Cruz focus on the evaluation of the spatial average of the throughput in a Poisson MANET using Spatial Aloha. The authors leverage the approach proposed in [1] to evaluate the optimal SINR target $T$ that a Poisson MANET using Spatial Aloha ought to use in order to maximize the throughput per unit area. The throughput is evaluated as the product of the probability of coverage and of the logarithm of $1+T$ (Shannon's capacity). However, this definition is only a lower bound to the real throughput obtained by transmitters: given that transmission is successful, namely given that the actual SINR $\theta$ at the receiver is larger than $T$, the throughput is the logarithm of $1+\theta$, which is larger than the logarithm of $1+T$ in general. The approach developed in $\S I I-D$ of the present paper tells us how much larger, since we actually compute the distribution and the mean of the logarithm of $1+\theta$. In this sense, the optimization of the density of throughput conducted in $\S \mathrm{V}$ of the present paper, which is one of our main new contributions, continues and refines that of [2].

In [3], Jacquet gives a closed form expression for the mean total throughput that a given receiver can obtain from a Poisson field of transmitters assuming that these emitters interfere with each other and that the throughput from a given emitter is defined, as in our model, as the logarithm of $1+$ SINR. In the Rayleigh fading case, he derives a closed form expression for the mean total throughput of such a communication model. The method is based on an evaluation of the mean area covered at a given SINR and on an elegant integration by part. The scheme which is proposed in the present paper is quite different: it is based on the $L_{2}$ isometry properties of the 
Fourier transform. This allows us to handle general fading distributions and to provide integral representations for the Laplace transform of the throughput and for its mean under general fading conditions.

An interesting special case when the distribution function of the interference can be given in the explicit form is presented in [4].

In [5], Hunter, Andrews and Weber focus on the evaluation of the performance of a Poisson MANET using Spatial Aloha together with single datastream MIMO techniques including beamforming, antenna sectorization and time-space block-coding techniques. The analysis builds upon the representation of the coverage probability based on shot noise processes which is recalled in Proposition 2.1 below. This line of thought will not be pursued here. Another important contribution of [5] consists in the analysis of the impact of certain fading scenarios such as e.g. Nakagami and Rayleigh. The new formalism based on the Fourier $L_{2}$ isometry which is developed in the present paper (Proposition 4.4 and Corollary 2.4) allows one to cope with arbitrary fading (like e.g. Rician beyond Nakagami and Rayleigh) and is expected to be a step forward in the understanding of this class of systems.

\section{Spatial Aloha in a Poisson MANET}

\section{A. The Poisson Bipolar Model with Independent Fading and Aloha MAC}

Below, we consider a Poisson bipolar network model in which each point of the Poisson pattern represents a node of the MANET and is hence a potential transmitter. Each node has an infinite backlog of packets to transmit and has an associated receiver located at distance $r$ (which is not part of the Poisson pattern of points). A natural extension that we will, however, not consider it in this paper consist in taking a random distance with some distribution of mean $r$.

More precisely, we assume that a snapshot of the MANET can be represented by an independently marked Poisson point process (P.p.p.) where the point process is homogeneous on the plane, with intensity $\lambda$ and where the multidimensional mark of a point carries information about the MAC status of the point (allowed to transmit or delayed) in the current time slot and about its fading conditions in the channels to all receivers. This marked P.p.p. will be denoted by $\widetilde{\Phi}=\left\{\left(X_{i}, e_{i}, y_{i}, \mathbf{F}_{i}\right)\right\}$, where

1) $\Phi=\left\{X_{i}\right\}$ denotes the locations of the points (the potential transmitters);

2) $\left\{e_{i}\right\}$ is the medium access indicator of node $i$; $\left(e_{i}=1\right.$ if node $i$ is allowed to transmit in the considered time slot and 0 otherwise). The random variables $e_{i}$ are hence i.i.d. and independent of everything else, with $\mathbf{P}\left(e_{i}=1\right)=p$.

3) $\left\{y_{i}\right\}$ denotes the location of the receiver for node $X_{i}$ (we assume here that no two transmitters have the same receiver). We assume that $\left\{X_{i}-y_{i}\right\}$ are i.i.d random vectors with $\left|X_{i}-y_{i}\right|=r$; i.e. each receiver is at distance $r$ from its transmitter (see Figure 1). There is no difficulty extending what is described below to the case where these distances are independent and identically distributed random variables, independent of everything else.

4) $\left\{\mathbf{F}_{i}=\left(F_{i}^{j}: j\right)\right\}$ where $F_{i}^{j}$ denotes the virtual power emitted by node $i$ (provided $e_{i}=1$ ) towards receiver $y_{j}$. By virtual power $F_{i}^{j}$, we understand the product of the effective power of transmitter $i$ and of the random fading from this node to receiver $y_{j}$. The random vectors $\left\{\mathbf{F}_{i}\right\}$ are assumed to be i.i.d., whereas the components $\left(F_{i}^{j}, j\right)$ are assumed to be identically distributed (distributed as a generic r.v. denoted by $F$ ) with mean $1 / \mu$ assumed finite. In the case of constant effective transmission power $1 / \mu$ and Rayleigh fading, $F$ is exponential with mean $1 / \mu$ (see e.g. [6, p. 50 and 501]). In this case it is reasonable to assume that the components of $\left(F_{i}^{j}: j\right)$ are independent, which will be the default option in what follows. This is justified if the distance between two receivers is larger than the coherence distance of the wireless channel (see [6]), which is a natural assumption here. Below, we will also consider non exponential cases which allow one to analyze other types of fading such as e.g. Rician or Nakagami scenarios or simply the case without fading (when $F \equiv 1 / \mu$ is deterministic).

In addition, we consider a nonnegative random variable $W$ independent of $\widetilde{\Phi}$ modeling the power of the external (thermal) noise. A natural extension consist in considering a random field rather than a random variable.

Note that the set of nodes that transmit in the reference time slot: $\Phi^{1}=\left\{X_{i}: e_{i}=1\right\}$ is a P.p.p. with intensity $\lambda_{1}=\lambda p$.

\section{B. Mean Path-loss Model}

Below, we assume that the receiver of node $i$ receives a power from the transmitter located at node $j$ which is equal to $F_{i}^{j} / l\left(\left|X_{j}-y_{i}\right|\right), F_{i}^{j}$ is the random fading from node $j$ to the receiver of node $i$ and where $|\cdot|$ denotes the Euclidean distance on the plane. In this formula, $l(\cdot)$ is the palth loss function. An important special case consists in taking

$$
l(u)=(A u)^{\beta} \text { for } A>0 \text { and } \beta>2 .
$$




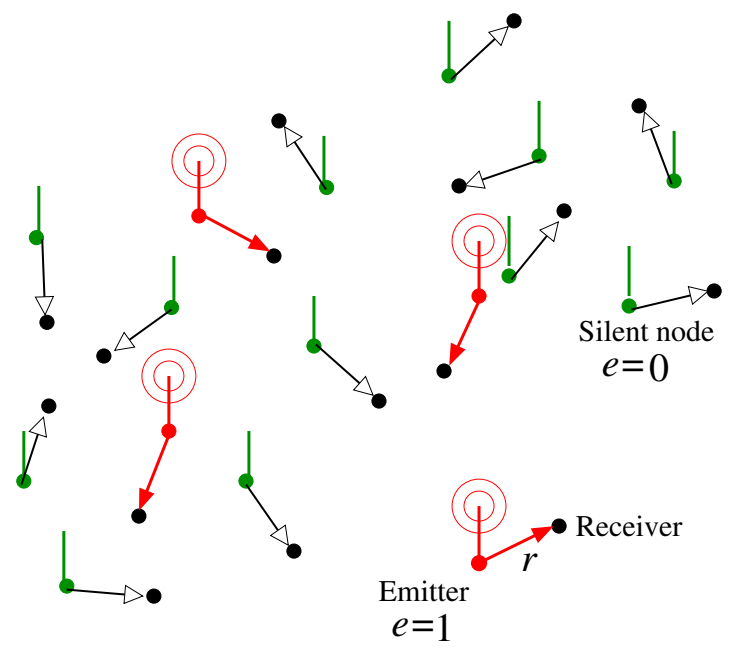

Fig. 1. A snapshot of bipolar MANET with Aloha MAC.

Note that $1 / l(u)$ has a pole at $u=0$, and thus in particular is not correct for small distances (and hence in particular for $u$ small compared to $1 / \sqrt{\lambda}$ ). Another inconvenience of this path-loss model is that the total power received at a given location from an infinite Poisson pattern of transmitters has an infinite mean (where averaging is taken over all configurations of transmitters).

Despite these drawbacks of the path-loss model (2.1), we will use it as our default model, because it is precise enough for large enough values of $u$, it simplifies many calculations and reveals important scaling laws (see Section II-E).

Other possible choices of path-loss function avoiding the pole at at $u=0$ consist in taking e.g. $\max (1, l(u))$, $l(u+1)$, or $l\left(\max \left(u, u_{0}\right)\right)$.

\section{Coverage (Non-Outage) Probability}

We will say that transmitter $\left\{X_{i}\right\}$ covers its receiver $y_{i}$ in the reference time slot if

$$
\operatorname{SINR}_{i}=\frac{F_{i}^{i} / l\left(\left|X_{i}-y_{i}\right|\right)}{W+I_{i}^{1}} \geq T,
$$

where $I_{i}^{1}$ is the shot-noise of $\widetilde{\Phi}^{1}$, namely, $I_{i}^{1}=$ $\sum_{X_{j} \in \widetilde{\Phi}^{1}, j \neq i} F_{j}^{i} / l\left(\left|X_{j}-y_{i}\right|\right)$, and where $T$ is some SINR threshold. We will equivalently say that $x_{i}$ can be successfully be received by $y_{i}$ or that $y_{i}$ is not in outage with respect to $x_{i}$ in the time slot.

Throughout the paper, we will use the following explicit formula for the Laplace transform of the generic shot-noise $I^{1}=\sum_{X_{j} \in \widetilde{\Phi}^{1}} F_{j} / l\left(\left|X_{j}\right|\right)$, which is valid in P.p.p. case whenever the random variables $F_{j}$ are independent copies of the generic fading variable $F$ :

$$
\begin{aligned}
\mathcal{L}_{I^{1}}(s) & =\mathbf{E}\left[e^{-I^{1} s}\right] \\
= & \exp \left\{-\lambda_{1} 2 \pi \int_{0}^{\infty} t\left(1-\mathcal{L}_{F}(s / l(t))\right) \mathrm{d} t\right\},
\end{aligned}
$$

where $\mathcal{L}_{F}$ is the Laplace transform of $F$. It can be derived from the formula for the Laplace functional of the Poisson p.p. (see e.g. [7]) and was already used in [1].

Denote by $\delta_{i}$ the indicator that (2.2) holds, namely, that location $y_{i}$ is covered by transmitter $X_{i}$ with the required quality. By the stationarity of $\widetilde{\Phi}$, the sequence $\left\{\delta_{i}\right\}$ is a stationary sequence of marks, which are however not independent.

Let $\mathbf{E}^{0}$ denote the Palm distribution of the P.p.p. Under this distribution, the nodes are located at $\widetilde{\Phi} \cup\left\{\left(X_{0}=\right.\right.$ $\left.\left.0, e_{0}, y_{0}, F_{0}\right)\right\}$, where $\left(e_{0}, y_{0}, \mathbf{F}_{0}\right)$ is independent of $\widetilde{\Phi}$, a stationary independently marked P.p.p., and is distributed like the other marks (cf. [8]). Note that under $\mathbf{E}^{0}$, the node at the origin, called the typical node, is not necessarily a transmitter; $e_{0}$ is equal to 1 or 0 with probability $p$ and $1-p$ respectively.

Denote by $p_{c}\left(r, \lambda_{1}, T\right)=\mathbf{E}^{0}\left[\delta_{0}\right]$ the probability that node $X_{0}=0$ covers its receiver, given it is a transmitter. Note that this probability depends in particular on the density of transmitters $\lambda_{1}=\lambda p$, the distance $r$ and the SINR threshold $T$, and that it can be expressed using three independent generic random variables $F, I^{1}, W$ by the following formula

$$
p_{c}\left(r, \lambda_{1}, T\right)=\mathbf{P}\left\{F \geq T l(r)\left(I^{1}+W\right)\right\} .
$$

Using the independence assumptions, it is easy to see that in the case of Rayleigh fading the last formula can be rewritten as

$$
\begin{aligned}
p_{c}(r, \lambda) & =\mathbf{E}\left[e^{-\mu\left(T l(r)\left(I^{1}+W\right)\right.}\right] \\
& =\mathcal{L}_{I^{1}}(\mu T l(r)) \mathcal{L}_{W}(\mu T l(r)),
\end{aligned}
$$

where $\mathcal{L}_{W}$ is the Laplace transform of $W$ and where $\mathcal{L}_{I^{1}}$ can be expressed from (2.3) when using the fact that $F$ is exponential:

$$
\mathcal{L}_{I^{1}}(s)=\exp \left(-2 \pi \lambda_{1} \int_{0}^{\infty} \frac{t}{1+\mu l(t) / s} \mathrm{~d} t\right) .
$$

Using this observation the following result was proven in [1].

Proposition 2.1: Assume the Poisson bipolar network model of Section II-A with Rayleigh fading ( $F$ exponential with mean $1 / \mu$ ). Then

$$
\begin{aligned}
p_{c}\left(r, \lambda_{1}, T\right)= & \mathcal{L}_{W}(\mu T l(r)) \\
& \times \exp \left\{-2 \pi \lambda_{1} \int_{0}^{\infty} \frac{u}{1+l(u) /(T l(r))} \mathrm{d} u\right\}
\end{aligned}
$$


In particular if $W \equiv 0$ and that the path-loss model (2.1) is used then

$$
p_{c}\left(r, \lambda_{1}, T\right)=\exp \left(-\lambda_{1} r^{2} T^{2 / \beta} K(\beta)\right),
$$

where

$$
K(\beta)=\frac{2 \pi \Gamma(2 / \beta) \Gamma(1-2 / \beta)}{\beta}=\frac{2 \pi^{2}}{\beta \sin (2 \pi / \beta)} .
$$

The above result can be used in the following context: assume one wants to operate a MANET in a regime where each transmitter is guaranteed a SINR at least $T$ with a probability larger than $1-\varepsilon$ where $\varepsilon$ is a predefined quality of service, or equivalently, where the probability of outage is less then $\varepsilon$. Then, if the transmitter-receiver distance is $r$, the MAP $p$ should be such that $p_{c}(r, \lambda p, T)=1-\varepsilon$. In particular, assuming the path-loss setting (2.1), one should take

$$
\begin{aligned}
p & =\min \left(1, \frac{-\ln (1-\varepsilon)}{\lambda r^{2} T^{2 / \beta} K(\beta)}\right) \\
& \approx \min \left(1, \frac{\varepsilon}{\lambda r^{2} T^{2 / \beta} K(\beta)}\right) .
\end{aligned}
$$

For example, for $T=10 \mathrm{~dB}^{5}, \beta=4, r=1$, one should take $p \approx \min (1,0.064 \varepsilon / \lambda)$.

In what follows, we consider the case of a general fading model. We show that under some additional regularity conditions one can get integral representations for the probability of coverage.

Proposition 2.2: Consider the Poisson bipolar network model of Section II-A with fading variables $F$ such that

- $F$ has a finite first moment and admits a square integrable density;

- Either $I^{1}$ or $W$ admit a density which is square integrable.

Then the probability of a successful transmission is equal to

$p_{c}\left(r, \lambda_{1}, T\right)$

$=\int_{-\infty}^{\infty} \mathcal{L}_{I^{1}}(2 i \pi l(r) T s) \mathcal{L}_{W}(2 i \pi l(r) T s) \frac{\mathcal{L}_{F}(-2 i \pi s)-1}{2 i \pi s} \mathrm{~d} s$

Remark: Sufficient conditions for $I^{1}$ to admit a density are given in [9, Prop. A.2]. Roughly speaking these conditions require non-null $F$ and the path-loss function $l$ is not constant in any interval. This is satisfied e.g. for $l(u)$ given by (2.1) and its modification $l(u+1)$, but not by the two other examples given at the end of Section II-B. Concerning the square integrability of the density, which is equivalent to the integrability of

\footnotetext{
${ }^{5} \mathrm{~A}$ positive real number $x$ is $10 \log _{10}(x) \mathrm{dB}$.
}

$\left|\mathcal{L}_{I^{1}}(i s)\right|^{2}$ (see [10, p.510]), using (2.3) one can easily check e.g. that it is satisfied for $l(u)$ given by (2.1) provided $\mathbf{P}\{F>0\}>0$. Moreover, under the same conditions $\left|\mathcal{L}_{I^{1}}(i s)\right|$ is integrable (and so is $\left|\mathcal{L}_{I^{1}}(i s)\right| /|s|$ for large $|s|)$.

Proof: (of Proposition 2.2) By the independence of $I^{1}$ and $W$, the second assumption of Proposition 2.2 implies that $I^{1}+W$ admits a density $g(\cdot)$ that is square integrable. Denote by $f(y)$ the (square integrable) density of $F$. From (2.4),

$$
\begin{aligned}
p_{c}\left(r, \lambda_{1}, T\right) & =\int_{0}^{\infty} f(y) \operatorname{Pr}\left(I^{1}+W<y /(T l(r))\right. \\
& =\int_{0}^{\infty} f(y) \int_{-\infty}^{\infty} g(t) \mathbb{I}(0<t<y /(T l(r)) \mathrm{d} t,
\end{aligned}
$$

so that by the Plancherel-Parseval theorem (see e.g. [11, Th. C3.3, p.157])

$$
\begin{gathered}
p_{c}\left(r, \lambda_{1}, T\right) \\
=\int_{0}^{\infty} f(y) \int_{-\infty}^{\infty} \mathcal{L}_{I^{1}}(-2 i \pi s) \mathcal{L}_{W}(-2 i \pi s) \\
\times \frac{e^{2 i \pi y s /(T l(r))}-1}{2 i \pi s} \mathrm{~d} s \mathrm{~d} y \\
=\int_{0}^{\infty} f(y) \int_{-\infty}^{\infty} \mathcal{L}_{I^{1}}(-2 i \pi s T l(r)) \mathcal{L}_{W}(-2 i \pi s T l(r)) \\
\times \frac{e^{2 i \pi y s}-1}{2 i \pi s} \mathrm{~d} s \mathrm{~d} y .
\end{gathered}
$$

The result follows provided one can interchange the two integrals. This is licit by Fubini's theorem provided

$$
\begin{aligned}
\int_{0}^{\infty} \int_{-\infty}^{\infty} f(y) \mid \mathcal{L}_{I^{1}}(-2 i \pi s T l & (r))|| \mathcal{L}_{W}(-2 i \pi s T l(r)) \mid \\
& \times\left|\frac{e^{2 i \pi y s}-1}{2 i \pi s}\right| \mathrm{d} s \mathrm{~d} y<\infty .
\end{aligned}
$$

By the second assumption of Proposition 2.2 either $\left|\mathcal{L}_{I^{1}}(i s)\right| /|s|$ or $\left|\mathcal{L}_{W}(i s)\right| /|s|$ is integrable for large $|s|$ (as a corollary of the Cauchy-Schwarz inequality). From this, we deduce that

$$
\left|\mathcal{L}_{I^{1}}(-2 i \pi s T l(r))\right|\left|\mathcal{L}_{W}(-2 i \pi s T l(r))\right|\left|\frac{e^{2 i \pi y s}-1}{2 i \pi s}\right|
$$

is integrable in $s$ for large $|s|$ and uniformly in $y$. For small $|s|$ this modulus is bounded from above by some constant $K$ times $y$ and the required absolute integrability holds because $F$ has finite first moment.

\section{Throughput}

Within the network scenario described above, one can also ask about the spatial average of the rates obtained by the transmitters. Assume the throughput of a channel with SINR $T$ is $\mathcal{T}=B \log (1+T)$ (we take $B=1$ to 


\begin{tabular}{|c|c|c|c|c|c|c|c|}
\hline & $r=.25$ & $r=.37$ & $r=.5$ & $r=.65$ & $r=.75$ & $r=.9$ & $r=1$ \\
\hline Rayleigh & 1.52 & .886 & .480 & .250 & .166 & .0930 & .0648 \\
\hline Erlang (8) & 1.71 & .942 & .495 & .242 & .155 & .0832 & .0571 \\
\hline
\end{tabular}

TABLE I

IMPACT OF THE FADING ON THE MEAN THROUGHPUT $\tau$ FOR VARYING DISTANCE $r$. ERLANG DISTRIBUTION OF ORDER 8 MIMICS NO-FADING CASE; $\beta=4$, EXPONENTIAL NOISE $W$ WITH MEAN 0.01 .

simplify notation). In what follows we are interested in the mean throughput of a typical node when it transmits, namely

$$
\tau\left(r, \lambda_{1}\right)=\mathbf{E}^{0}\left[\mathcal{T}_{0}\right]=\mathbf{E}^{0}\left[\log \left(1+\operatorname{SINR}_{0}\right)\right]
$$

and also its Laplace transform

$$
\mathcal{L}_{\mathcal{T}}(s)=\mathbf{E}^{0}\left[e^{-s \mathcal{T}_{0}}\right]=\mathbf{E}^{0}\left[\left(1+\operatorname{SINR}_{0}\right)^{-s}\right] .
$$

Let us now make the following simple observations:

$$
\begin{aligned}
\mathbf{E}^{0}[ & \left.\log \left(1+\operatorname{SINR}_{0}\right)\right] \\
& =\int_{0}^{\infty} \mathbf{P}^{0}\left\{\log \left(1+\operatorname{SINR}_{0}\right)>t\right\} \mathrm{d} t \\
& =\int_{0}^{\infty} \mathbf{P}^{0}\left\{\operatorname{SINR}_{0}>e^{t}-1\right\} \mathrm{d} t \\
& =\int_{0}^{\infty} p_{c}\left(r, \lambda_{1}, e^{t}-1\right) \mathrm{d} t
\end{aligned}
$$

and similarly

$$
\mathbf{E}^{0}\left[\left(1+\mathrm{SINR}_{0}\right)^{-s}\right]=1-\int_{0}^{1} p_{c}\left(r, \lambda_{1}, t^{-1 / s}-1\right) \mathrm{d} t
$$

provided $\mathbf{P}\left\{\operatorname{SINR}_{0}=T\right\}=0$ for all $T \geq 0$, which is true e.g. when $F$ admits a density (cf. (2.4)). This in conjunction with Propositions 2.1 and 2.2 leads to the following results:

Corollary 2.3: Under the assumptions of Proposition 2.1 (namely for the model with Rayleigh fading) and for the path-loss model (2.1)

$$
\tau=\frac{\beta}{2} \int_{0}^{\infty} e^{-\lambda_{1} K(\beta) r^{2} v} \frac{v^{\frac{\beta}{2}-1}}{1+v^{\frac{\beta}{2}}} \mathcal{L}_{W}\left(\mu(A r)^{\beta} v^{\beta / 2}\right) \mathrm{d} v
$$

and

$$
\begin{gathered}
\mathcal{L}_{\mathcal{T}}(s) \\
=\frac{\beta s}{2} \int_{0}^{\infty}\left(1-e^{-\lambda_{1} K(\beta) r^{2} v} \mathcal{L}_{W}\left(\mu(A r)^{\beta} v^{\beta / 2}\right)\right) \\
\times \frac{v^{\frac{\beta}{2}-1}}{\left(1+v^{\frac{\beta}{2}}\right)^{1+s}} \mathrm{~d} v
\end{gathered}
$$

where $K(\beta)$ is defined in (2.9).

Corollary 2.4: Under the assumptions of Proposi- tion 2.2 (namely in the model with general fading $F$ )

$$
\begin{aligned}
\tau= & \int_{0}^{\infty} \int_{-\infty}^{\infty} \mathcal{L}_{I^{1}}\left(2 i \pi l(r)\left(e^{t}-1\right) s\right) \\
& \times \mathcal{L}_{W}\left(2 i \pi l(r)\left(e^{t}-1\right) s\right) \frac{\mathcal{L}_{F}(-2 i \pi s)-1}{2 i \pi s} \mathrm{~d} s \mathrm{~d} t
\end{aligned}
$$

and

$$
\begin{aligned}
& \mathcal{L}_{\mathcal{T}}(s) \\
& =1-\int_{0}^{1} \int_{-\infty}^{\infty} \mathcal{L}_{I^{1}}\left(2 i \pi l(r)\left(t^{-1 / s}-1\right) s\right) \\
& \times \mathcal{L}_{W}\left(2 i \pi l(r)\left(t^{-1 / s}-1\right) s\right) \frac{\mathcal{L}_{F}(-2 i \pi s)-1}{2 i \pi s} \mathrm{~d} s \mathrm{~d} t .
\end{aligned}
$$

Here is a direct application of the last results. Table I shows how Rayleigh fading compares to the situation with no fading. The path-loss model (2.1) is assumed with $A=1$ and $\beta=4$. We assume $W$ to be exponential with mean 0.01 . We use the formulas of the last corollaries; the Rayleigh case is with $F$ exponential of parameter 1 ; to represent the no fading case within this framework we take $F$ Erlang of high order (here 8) with the same mean 1 as the exponential. We see that the presence of fading is beneficial in the far-field, and detrimental in the near-field.

\section{E. Scaling Properties}

We show below that in the Poisson bipolar network model of Section II-A, when using the path-loss model (2.1) and when $W=0$, some interesting scaling properties can be derived.

Denote by $\bar{p}_{c}(r)=p_{c}(r, 1, T)$ the value of the probability of connection calculated in this model with $T \equiv 1, \lambda_{1}=1, W \equiv 0$ and normalized virtual powers $\bar{F}_{i}^{j}=\mu F_{i}^{j}$. Note that $\bar{p}_{c}(r)$ does not depend on any parameter of the model other than the distribution of the normalized virtual power $\bar{F}$.

Proposition 2.5: In the Poisson bipolar network model of Section II-A with path-loss model (2.1) and $W=0$

$$
p_{c}\left(r, \lambda_{1}, T\right)=\bar{p}_{c}\left(r T^{1 / \beta} \sqrt{\lambda_{1}}\right) .
$$

Proof: The Poisson point process $\Phi^{1}$ with intensity $\lambda_{1}>0$ can be represented as $\left\{X_{i}^{\prime} / \sqrt{\lambda_{1}}\right\}$, where 
$\Phi^{\prime}=\left\{X_{i}^{\prime}\right\}$ is Poisson with intensity 1 . Because of this, under (2.1), the Poisson shot-noise interference variable $I^{1}$ admits the following representation: $I^{1}=\lambda_{1}^{\beta / 2} I^{1}$, where $I^{\prime 1}$ is defined in the same manner as $I^{1}$ but with respect to $\Phi^{\prime}$. Thus for $W=0$,

$$
\begin{aligned}
p_{c}\left(r, \lambda_{1}, T\right) & =\mathbf{P}\left(F \geq T(A r)^{\beta} I^{1}\right) \\
& =\mathbf{P}\left(\mu F \geq \mu\left(A r T^{1 / \beta} \lambda_{1}^{1 / 2}\right)^{\beta} I^{\prime 1}\right) \\
& =\bar{p}_{c}\left(r T^{1 / \beta} \sqrt{\lambda_{1}}\right) .
\end{aligned}
$$

\section{Some Spatial Performance Metrics}

The knowledge of $p_{c}\left(r, \lambda_{1}, T\right)$ or of $\tau\left(r, \lambda_{1}\right)$ allows one to estimate the following performance metrics:

- The (spatial) density of successful transmissions is defined as the mean number of successful transmissions per unit area:

$$
d_{\text {suc }}\left(r, \lambda_{1} \cdot T\right)=\frac{1}{|B|} \mathbf{E}\left[\sum_{i} e_{i} \delta_{i} \mathbb{I}\left(X_{i} \in B\right)\right] .
$$

From stationarity, the last definition does not depend on the particular choice of Borel set $B$. By Campbell's formula

$$
d_{\text {suc }}\left(r, \lambda_{1} . T\right)=\lambda_{1} p_{c}\left(r, \lambda_{1}, T\right)=\lambda p p_{c}(r, \lambda p, T) .
$$

- The mean progress made in a typical transmission, defined as

$$
\operatorname{prog}\left(r, \lambda_{1}, T\right)=r \mathbf{E}^{0}\left[\delta_{0}\right]=r p_{c}\left(r, \lambda_{1}, T\right) .
$$

- The (spatial) density of progress, defined as the mean number of meters progressed by all transmissions taking place per unit surface area:

$$
\begin{aligned}
d_{\text {prog }}\left(r, \lambda_{1}, T\right) & =\frac{1}{|B|} \mathbf{E}\left[\sum_{i} r e_{i} \delta_{i} \mathbb{I}\left(X_{i} \in B\right)\right] \\
& =r \lambda_{1} p_{c}\left(r, \lambda_{1}, T\right)
\end{aligned}
$$

by the same arguments as for (3.1).

- The (spatial) density of throughput is defined as the mean throughput per unit surface area:

$$
\begin{aligned}
& d_{\text {throu }}\left(r, \lambda_{1}\right) \\
& \quad=\frac{1}{|B|} \mathbf{E}\left[\sum_{i} e_{i} \mathbb{I}\left(X_{i} \in B\right) \log \left(1+\operatorname{SINR}_{i}\right)\right] \\
& \quad=\lambda_{1} \tau\left(r, \lambda_{1}\right) .
\end{aligned}
$$

- The (spatial) density of transport

$$
\begin{aligned}
& d_{\text {trans }}\left(r, \lambda_{1}\right) \\
& \quad=\frac{1}{|B|} \mathbf{E}\left[\sum_{i} e_{i} r \mathbb{I}\left(X_{i} \in B\right) \log \left(1+\operatorname{SINR}_{i}\right)\right] \\
& \quad=\lambda_{1} r \tau\left(r, \lambda_{1}\right) .
\end{aligned}
$$

This is the mean number of bit-meters transported per second per unit and space.

In the following sections we will be interested in optimizing the spatial performance of an Aloha MANET.

\section{Optimization of the Density of Progress}

\section{A. Best MAP Given Some Transmission Distance}

We mentioned in the introduction that a good tuning of $p$ should find a compromise between the average number of concurrent transmissions per unit area and the probability that a given authorized transmission will be successful. To find such a compromise, one ought to maximize the density of progress, or equivalently the density of successful transmissions, $d_{\text {suc }}(r, \lambda p, T)=$ $\lambda p p_{c}(r, \lambda p, T)$, w.r.t. $p$, for a given $r$ and $\lambda$. As already shown in [1] this can be done explicitly for the Poisson bipolar network model with Rayleigh fading.

Define

$$
\lambda_{\max }=\underset{0 \leq \lambda<\infty}{\arg \max } d_{\text {suc }}(r, \lambda, T)
$$

whenever such a value of $\lambda$ exists and is unique. The following result was proved in [1].

Proposition 4.1: Under the assumptions of Proposition 2.1 (in the model with Rayleigh fading) with $p=1$ the unique maximum of the density of successful transmissions $d_{\text {suc }}(r, \lambda, T)$ is attained at

$$
\lambda_{\max }=\left(2 \pi \int_{0}^{\infty} \frac{u}{1+l(u) /(T l(r))} \mathrm{d} u\right)^{-1},
$$

and the maximal value is equal to

$$
d_{\text {suc }}\left(r, \lambda_{\max }, T\right)=e^{-1} \lambda_{\max } \mathcal{L}_{W}(\mu T l(r)) .
$$

In particular, assuming $W \equiv 0$ and path-loss (2.1)

$$
\begin{aligned}
\lambda_{\max } & =\frac{1}{K(\beta) r^{2} T^{2 / \beta}}, \\
d_{\text {suc }}\left(r, \lambda_{\max }, T\right) & =\frac{1}{e K(\beta) r^{2} T^{2 / \beta}} .
\end{aligned}
$$

with $K(\beta)$ defined in (2.9).

The above result yields the following corollary concerning the tuning of the MAC parameter when $\lambda$ is fixed.

Corollary 4.2: Under assumptions of Proposition 2.1 (in the model with Rayleigh fading) with some given $r$ 
the value of the MAP $p$ that maximizes the density of successful transmissions is

$$
p_{\max }=\min \left(1, \lambda_{\max } / \lambda\right) .
$$

In order to extend our observations to general fading (or equivalently to a general distribution for virtual power) let us assume that $W=0$ and that the path-loss function is (2.1). Then, using Lemma 2.5, we can easily show that $\lambda_{\max }$ and $d_{\text {suc }}\left(r, \lambda_{\max }\right)$, exhibit, up to some constant, the same dependence on the model parameters, namely the distance $r$ from transmitter to receiver, $T$ the threshold and $\mu$ the inverse of the mean of virtual power $F$, as that given in (4.1) and (4.2) for exponential $F$.

Proposition 4.3: In the Poisson bipolar network model of Section II-A with general fading, with pathloss model (2.1) and $W=0$

$$
\begin{aligned}
\lambda_{\max } & =\frac{c_{1}}{r^{2} T^{2 / \beta}}, \\
d_{\text {suc }}\left(r, \lambda_{\max }\right) & =\frac{c_{2}}{r^{2} T^{2 / \beta}},
\end{aligned}
$$

where the constants $c_{1}$ and $c_{2}$ do not depend on $r, T, \mu$, provided $\lambda_{\max }$ is well defined.

Proof: Assume that $\lambda_{\max }$ is well defined. By Lemma 2.5, $c_{1}=\arg \max _{\lambda \geq 0}\left\{\lambda \bar{p}_{c}(\sqrt{\lambda})\right\}$ and $c_{2}=$ $\max _{\lambda \geq 0}\left\{\lambda \bar{p}_{c}(\sqrt{\lambda})\right\}$.

So the main question is that of the definition of $\lambda_{\max }$ which is addressed below.

\section{B. General Definition of $\lambda_{\max }$}

In this section, we show that under some mild conditions, $\lambda_{\max }$ is well defined and not degenerate (i.e. $0<$ $\left.\lambda_{\max }<\infty\right)$. Assume $T>0$. Note that $d_{\text {suc }}(r, 0, T)=0$; so under some natural non-degeneracy assumptions, the maximum is certainly not attained at $\lambda=0$.

Proposition 4.4: Consider the Poisson bipolar network model of Section II-A with $p=1$ and general fading with a finite mean. Assume that $l(r)>0$ and is such that the generic shot-noise $I(\lambda)=\sum_{X_{j} \in \widetilde{\Phi}} F_{j} / l\left(\left|X_{j}\right|\right)$ admits a density for all $\lambda>0$. Then

1) If $\mathbf{P}\{F>0\}>0$, then $p_{c}(r, x, T)$ (and so $\left.d_{\text {suc }}(r, x, T)\right)$ is continuous in $x$, so that the maximum of the function $x \rightarrow d_{\text {suc }}(r, x, T)$ in the interval $[0, \lambda]$ is attained for some $0<\lambda_{\max } \leq \lambda$;

2) If for all $a>0$, the modified shot-noise at the origin:

$$
I^{\prime}(\lambda)=\sum_{X_{j} \in \widetilde{\Phi}} \mathbb{I}\left(\left|X_{j}\right|>a\right) F_{j} / l\left(\left|X_{j}\right|\right)
$$

has finite mean for all $\lambda>0$, then $\lim _{x \rightarrow \infty} d_{\text {suc }}(r, x, T)=0$ and consequently, for sufficiently large $\lambda$, this maximum is attained for some $\lambda_{\max }<\lambda$.

The statement of the last theorem means that for a sufficiently large density of nodes $\lambda$, a nontrivial MAP $0<p_{\max }<1$ equal to $p_{\max }=\lambda_{\max } / \lambda$ will optimize the density of successful transmissions.

Proof: (of Proposition 4.4) Recall that $p_{c}(r, \lambda, T)=$ $\mathbf{P}\{I(\lambda) \leq F /(l(r) T-W)\}$, where we made the dependence of the shot-noise variable $I(\lambda)=I^{1}=I$ (note that $p=1$ ) on the intensity of the Poisson p.p. explicit. By the thinning property of the Poisson p.p. we can split the shot-noise variable into two independent Poisson shot-noise terms $I(\lambda+\epsilon)=I(\lambda)+I(\epsilon)$. Moreover, we can do this in such a way that $I(\epsilon)$, which is finite by assumption, almost surely converges to 0 when $\epsilon \rightarrow 0$. Consequently,

$$
\begin{aligned}
0 & \leq p_{c}(r, \lambda, T)-p_{c}(r, \lambda+\epsilon, T) \\
& =\mathbf{P}\left\{\frac{F}{l(r) T-W}-I(\epsilon)<I(\lambda) \leq \frac{F}{l(r) T-W}\right\}
\end{aligned}
$$

and

$$
\begin{aligned}
& \lim _{\epsilon \rightarrow 0}\left(p_{c}(r, \lambda, T)-p_{c}(r, \lambda+\epsilon, T)\right) \\
& \quad=\mathbf{P}\left\{I(\lambda)=\frac{F}{l(r) T-W}\right\}=0,
\end{aligned}
$$

where the last equation is due to the fact that $I(\lambda)$ is independent of $F, W$ and admits a density. Splitting the P.p.p of intensity $\lambda$ into two P.p.p's with intensity $\lambda-\epsilon$ and $\epsilon$, and considering the associated shot-noise variables $I(\lambda-\epsilon)$ and $I(\epsilon)$, with $I(\lambda)$ defined as their sum, one can show in a similar manner that $\lim _{\epsilon \rightarrow 0} p_{c}(r, \lambda-\epsilon, T)-$ $p_{c}(r, \lambda, T)=0$. This concludes the proof of the first part of the proposition.

We now prove the second part. Let $\bar{G}(s)=\mathbf{P}\{F \geq$ $s\}$. Take $\epsilon>0$ and such that $\epsilon<\mathbf{E}\left[I^{\prime}(1)\right]=\bar{I}^{\prime}(1)<\infty$. By independence we have

$$
\begin{aligned}
& d_{\text {suc }}(r, \lambda, T) \\
& \quad=\lambda p_{c}(r, \lambda, T) \leq \lambda \mathbf{E}\left[\mathbf{P}\left\{F \geq I^{\prime}(\lambda) T l(r) \mid I^{\prime}(\lambda)\right\}\right] \\
& \quad \leq J_{1}+J_{2},
\end{aligned}
$$

where

$$
\begin{aligned}
& J_{1}= \mathbf{E}\left[\frac{\lambda}{I^{\prime}(\lambda)} \mathbb{I}\left(I^{\prime}(\lambda) \geq \lambda\left(\bar{I}^{\prime}(1)-\epsilon\right)\right)\right. \\
&\left.\times I^{\prime}(\lambda) \bar{G}\left(I^{\prime}(\lambda) T l(r)\right)\right] \\
& J_{2}=\lambda \mathbf{E}\left[\mathbb{I}\left(I^{\prime}(\lambda)<\lambda\left(\bar{I}^{\prime}(1)-\epsilon\right)\right)\right] .
\end{aligned}
$$

Since $\mathbf{E}[F]=\int_{0}^{\infty} \bar{G}(s) \mathrm{d} s<\infty, I^{\prime}(\lambda) \bar{G}\left(I^{\prime}(\lambda) T l(r)\right)$ is uniformly bounded in $I^{\prime}(\lambda)$ and $I^{\prime}(\lambda) \bar{G}\left(I^{\prime}(\lambda) T l(r)\right) \rightarrow$ 0 when $I^{\prime}(\lambda) \rightarrow \infty$. Moreover, one can construct a 
probability space such that $I^{\prime}(\lambda) \rightarrow \infty$ almost surely as $\lambda \rightarrow \infty$. Thus, by Lebesgue's dominated convergence theorem we have $\lim _{\lambda \rightarrow \infty} J_{1}=0$.

For $J_{2}$ and $t>0$ we have

$$
\begin{aligned}
J_{2} & \leq \lambda \mathbf{P}^{0}\left\{e^{-t I^{\prime}(\lambda)} \geq e^{-\lambda t\left(\bar{I}^{\prime}(1)-\epsilon\right)}\right\} \\
\leq & \lambda \mathbf{E}^{0}\left[e^{-t I^{\prime}(\lambda)+\lambda t\left(\bar{I}^{\prime}(1)-\epsilon\right)}\right] \\
& =\lambda \exp \left\{\lambda \left(t\left(\bar{I}^{\prime}(1)-\epsilon\right)\right.\right. \\
& \left.\left.\quad-2 \pi \int_{a}^{\infty} s\left(1-\mathcal{L}_{F}(t / l(s))\right) \mathrm{d} s\right)\right\} .
\end{aligned}
$$

Note that the derivative of $t\left(\bar{I}^{\prime}(1)-\epsilon\right)-2 \pi \int_{a}^{\infty} s(1-$ $\left.\mathcal{L}_{F}(t / l(s))\right) \mathrm{d} s$ with respect to $t$ at $t=0$ is equal to $\bar{I}^{\prime}(1)-\epsilon-\bar{I}^{\prime}(1)<0$. Thus, for some small $t>0$, $J_{2} \leq \lambda e^{-\lambda C}$ for some constant $C>0$. This shows that $\lim _{\lambda \rightarrow \infty} J_{2}=0$, which concludes the proof.

\section{Best Transmission Distance Given Some Transmitter Density}

Assume now some given intensity $\lambda_{1}$ of transmitters. We look for the distance $r$ which maximizes the mean density of progress, or equivalently the mean progress $\operatorname{prog}\left(r, \lambda_{1}, T\right)=r p_{c}\left(r, \lambda_{1}, T\right)$. We denote by

$$
r_{\max }=r_{\max }(\lambda)=\underset{r \geq 0}{\arg \max } \operatorname{prog}(r, \lambda, T)
$$

the best transmission distance for the density of transmitters $\lambda$ whenever such a value exists and is unique. Let

$$
\rho=\rho(\lambda)=\operatorname{prog}\left(r_{\max }(\lambda), \lambda, T\right)
$$

be the optimal mean progress.

Proposition 4.5: In the Poisson bipolar network model of Section II-A with general fading, with pathloss model (2.1) and $W=0$

$$
\begin{aligned}
r_{\max }(\lambda) & =\frac{c_{3}}{T^{1 / \beta} \sqrt{\lambda}}, \\
\rho(\lambda) & =\frac{c_{4}}{T^{1 / \beta} \sqrt{\lambda}},
\end{aligned}
$$

where the constants $c_{3}$ and $c_{4}$ do not depend on $R, T, \mu$, provided $r_{\max }$ is well defined. If $F$ is exponential (i.e. for Rayleigh fading) and $l(r)$ given by (2.1) then $c_{3}=$ $1 / \sqrt{2 K(\beta)}$ and $c_{4}=1 / \sqrt{2 e K(\beta)}$.

Proof: The result for general fading follows from Lemma 2.5. The constants for the exponential case can be evaluated by (2.8).

Remark: We see that the optimal distance $r_{\max }(\lambda)$ from transmitter to receiver is of the order of the distance to the nearest neighbor of the transmitter, namely $1 /(2 \sqrt{\lambda})$. Notice also that for Rayleigh fading and $l(r)$ given by (2.1) we have the general relation:

$$
2 r^{2} \lambda_{\max }(r)=r_{\max }(\lambda)^{2} \lambda .
$$

As before, one can show that for a general model, under some regularity conditions, $\operatorname{prog}(r, \lambda, T)$ is continuous in $r$ and that the maximal mean progress is attained for some positive and finite $r$. We skip these technicalities.

\section{Degeneracy of Two Step Optimization}

Assume for simplicity a $W=0$ and path-loss (2.1). In Section IV-A, we found that for fixed $r$, the optimal density of successful transmissions $d_{\text {suc }}$ is attained when the density of transmitters is equal to $\lambda_{1}=\lambda_{\max }=$ $c_{1} /\left(r^{2} T^{2 / \beta}\right)$. It is now natural to look for the distance $r$ maximizing the mean progress for the network with this optimal density of transmitters. But by Proposition 4.3

$$
\begin{aligned}
\sup _{r \geq 0} \operatorname{prog}\left(r, \lambda_{\max }, T\right) & =\sup _{r \geq 0} r p_{c}\left(r, \lambda_{\max }, T\right) \\
& =\sup _{r \geq 0} r \frac{d_{s u c}\left(r, \lambda_{\max }, T\right)}{\lambda_{\max }} \\
& =\sup _{r \geq 0} r \frac{c_{2}}{c 1}=\infty
\end{aligned}
$$

and thus the optimal choice of $r$ consists in taking $r=$ $\infty$, and consequently $\lambda_{\max }=0$. From a practical point of view, this is not of course an acceptable answer. Even if $r=\infty$ might be a consequence of taking $W=0$ the above observation might suggest that for a small $W>0$ a (possibly) finite optimal value of $r$ would be too large from a practical point of view. In a network-perspective, one might better optimize a more "social" characteristic of the MANET like e.g. the density of progress $d_{\text {prog }}=$ $\lambda r p_{c}(r, \lambda, T)$ first in $\lambda$ and then in $r$. However in this case one obtains the opposite degenerate answer:

$$
\begin{aligned}
\sup _{r \geq 0} d_{\text {prog }}\left(r, \lambda_{\max }, T\right) & =\sup _{r \geq 0} r d_{\text {suc }}\left(r, \lambda_{\max }, T\right) \\
& =\sup _{r \geq 0} r \frac{c_{2}}{r^{2} T^{2 / \beta}}=0,
\end{aligned}
$$

which is attained for $r=0$ and $\lambda_{\max }=\infty$.

The above analysis shows that a better receiver model of Aloha MANET is needed to study the joint optimization in $r$ and in $\lambda$. Such a model, proposed in the second part of [1], assumes that the receivers are no longer sampled as independent marks of the Poisson p.p. of potential transmitters, but belong to the point process of potential transmitters and are chosen amongst the nodes from which the Aloha mechanism prevents transmission during the time slot considered. However, due to space limitations, in the remaining part of the present paper 
we will not go beyond the bipolar network model of Section II-A.

\section{Optimization of the Density of TRANSPoRT}

\section{A. Best MAP Given Some Transmission Distance}

Define

$$
\lambda_{\max }^{\tau}=\underset{0 \leq \lambda<\infty}{\arg \max } d_{\text {trans }}(r, \lambda)
$$

whenever such a value of $\lambda$ exists and is unique. We have the following result.

Proposition 5.1: In the Poisson bipolar network model of Section II-A with Rayleigh fading, path-loss model (2.1) and $W=0$, the unique maximum $\lambda_{\max }^{\tau}$ of the density of transport $d_{\text {trans }}(r, \lambda)$ is attained at

$$
\lambda_{\max }^{\tau}=\frac{x^{*}(\beta)}{r^{2} K(\beta)}
$$

where $x^{*}(\beta)$ is the unique solution of the integral equation

$$
\int_{0}^{\infty} e^{-x v} \frac{v^{\frac{\beta}{2}-1}}{1+v^{\frac{\beta}{2}}} \mathrm{~d} v=x \int_{0}^{\infty} e^{-x v} \frac{v^{\frac{\beta}{2}}}{1+v^{\frac{\beta}{2}}} \mathrm{~d} v .
$$

Proof: One obtains this characterization by differentiating (2.13) w.r.t. $\lambda_{1}$.

\section{B. Best Transmission Distance Given a Density of Trans- mitters}

Assume now some given intensity $\lambda_{1}=\lambda p$ of transmitters. We look for the distance $r$ which maximizes the mean density of transport, or equivalently the mean throughput $\tau\left(r, \lambda_{1}\right)$. We denote by

$$
r_{\max }^{\tau}=r_{\max }^{\tau}(\lambda)=\underset{r \geq 0}{\arg \max } r \tau(r, \lambda)
$$

the best transmission distance for this criterion.

Proposition 5.2: In the Poisson bipolar network model of Section II-A with Rayleigh fading, path-loss model (2.1) and $W=0$, the unique maximum of the density of transport $d_{\text {trans }}(r, \lambda)$ is attained at

$$
r_{\max }^{\tau}=\sqrt{\frac{y^{*}(\beta)}{\lambda K(\beta)}},
$$

where $y^{*}(\beta)$ is the unique solution of the integral equation

$$
\int_{0}^{\infty} e^{-y v} \frac{v^{\frac{\beta}{2}-1}}{1+v^{\frac{\beta}{2}}} \mathrm{~d} v=2 y \int_{0}^{\infty} e^{-y v} \frac{v^{\frac{\beta}{2}}}{1+v^{\frac{\beta}{2}}} \mathrm{~d} v .
$$

Proof: One obtains this characterization by differentiating (2.13) w.r.t. $r$.

We will not pursue this line of thought any further. Let us nevertheless point out that the last results can be extended to more general fading models and also that the same degeneracies as those mentioned above take place.

\section{Vi. Spatial Reuse in Optimized Poisson MANETS}

Aloha creates a random exclusion disc around each transmitter. By this we mean that for an arbitrary radius there is some non-null probability that all the nodes in the disk with this radius do not transmit at a given time slot. Below we evaluate the mean exclusion radius and for comparison we consider an equivalent CSMA model with exclusion radius equal to this mean. Note, that in this latter model, the exclusion disk is deterministic. Specifically, CSMA uses a carrier sense threshold above which a node defers its transmission. This creates an exclusion disc around the transmitter where no concurrent transmission can take place.

We define the mean exclusion radius of a Poisson MANET using Aloha as the mean distance from a typical transmitter to its nearest concurrent transmitter

$$
R_{e x c l}=\mathbf{E}^{0}\left[\min _{i \neq 0}\left\{\left|X_{i}\right|: e_{i}=1\right\}\right] .
$$

For the Poisson network this means that the exclusion radius is equal to

$$
R_{e x c l}=R_{e x c l}\left(\lambda_{1}\right)=\frac{1}{2 \sqrt{\lambda_{1}}}=\frac{1}{2 \sqrt{\lambda p}} .
$$

Here are two questions pertaining to an optimized scenario and which can be answered using the results of the previous sections:

- If $r$ is given and $p$ is optimized, how does the resulting $R_{\text {excl }}$ compare to $r$ ?

- If $\lambda$ is given and $r$ is optimized, how does the resulting $r$ compare to $R_{e x c l}$ ?

We will in fact address these questions in a unified way by defining the spatial reuse factor of the Poisson MANET as the ratio of the typical distance $r$ between transmitter and receiver and of the typical distance between nearest neighbor transmitters (this notion is analogous to the concept of spectral reuse in cellular networks). So if the spatial density of transmitters in this Aloha MANET is $\lambda_{1}$, then

$$
S_{\text {reuse }}=S_{\text {reuse }}\left(\lambda_{1}, r\right)=\frac{r}{R_{\text {excl }}}=2 r \sqrt{\lambda_{1}} .
$$

Here are a few illustrations.

Example 6.1: Consider the Poisson bipolar network model of Section II-A. Assume general fading, that the path-loss model is (2.1) and that $W=0$. Assume that the SINR target is $T$ and that the transmitter-receiver distance is $r$. We deduce from Proposition 4.3 that the 
spatial intensity of transmitters that maximises the density of successful transmissions is $\lambda_{\max }=c_{1} /\left(r^{2} T^{2 / \beta}\right)$. Hence by (6.1)

$$
R_{\text {excl }}\left(\lambda_{\max }\right)=\frac{1}{2 \sqrt{\lambda_{\max }}}=r \frac{T^{1 / \beta}}{2 \sqrt{c_{1}}},
$$

so that at the optimum, the spatial reuse

$$
S_{\text {reuse }}=\frac{2 \sqrt{c_{1}}}{T^{1 / \beta}},
$$

is independent of $r$. For example, for $\beta=4$ and Rayleigh fading, we can use the fact that $c_{1}=1 / K(\beta)$ to evaluate the last expressions. For a SINR target of $T=10 \mathrm{~dB}$, $R_{\text {excl }}\left(\lambda_{\max }\right) \approx 1.976 r$. Equivalently $S_{\text {reuse }} \approx 0.506$. In order to have a spatial reuse larger than 1 , one needs a SINR target less than $(2 \sqrt{2} / \pi)^{4}=0.657$, that is less than $-1.82 \mathrm{~dB}$.

Example 6.2: Consider the Poisson bipolar network model of Section II-A with general fading, path-loss model (2.1) and $W=0$ and target SINR T. Assume that the spatial density of transmitters is fixed and equal to $\lambda$. Let $r_{\max }(\lambda)$ denote the transmitter-receiver distance which maximizes the mean progress. We get from Proposition 4.5 that at the optimum $r$,

$$
S_{\text {reuse }}=\frac{c_{3}}{2 T^{1 / \beta}},
$$

for all values of $\lambda$. For $\beta=4$ and Rayleigh fading, if we pick a SINR target of $10 \mathrm{~dB}$, then $S_{\text {reuse }} \approx 0.358$ only. Similarly, $S_{\text {reuse }}>1$ iff $T<(2 / K(\beta))^{\beta / 2}$. For $\beta=4$, this is iff $T<0.164$ or equivalently $T$ less than -7.84 $\mathrm{dB}$.

Example 6.3: Assume the Poisson bipolar network model of Section II-A with Rayleigh fading, path-loss model (2.1) and $W=0$. Assume that the target distance between transmitter and receiver is $r$. We deduce from Proposition 5.1 that in terms of density of throughput, the best organization of the MANET is that where the spatial intensity of transmitters is $\lambda_{\max }^{\tau}=x(\beta) / r^{2} K(\beta)$. Hence, at the optimum,

$$
R_{e x c l}=r \frac{1}{2} \sqrt{\frac{K(\beta)}{x^{*}(\beta)}},
$$

so that

$$
S_{\text {reuse }}=2 \sqrt{\frac{x^{*}(\beta)}{K(\beta)}},
$$

a quantity that again does not depend on $r$. For $\beta=4$, one gets $x^{*}(\beta) \approx 0.771$, so that $R_{\text {excl }} \approx 1.27 r$ and $S_{\text {reuse }} \approx 0.790$.

Example 6.4: Consider the same scenario as in the last example but assume now that the intensity of transmitter is $\lambda$ fixed. Proposition 5.2 shows that in order to maximize the mean throughput, the optimal scenario is one where the transmitter-receivers distance $r_{\max }^{\tau}$ is such that

$$
S_{\text {reuse }}=2 \sqrt{\frac{y^{*}(\beta)}{K(\beta)}} .
$$

For $\beta=4, y^{*}(\beta) \approx 0.122$ and $S_{\text {reuse }} \approx 0.314$.

\section{Opportunistic AlOHA}

\section{A. Definition}

In the basic Spatial Aloha scheme, each node tosses a coin to access the medium independently of the fading variables. It is clear that something more clever can be done by combining the random selection of transmitters with the occurrence of good channel conditions. The general idea of Opportunistic Aloha is to select the nodes with a $F_{i}^{i}$ larger than a certain threshold as transmitters in the reference time slot. This threshold may be deterministic or random (we assume fading variables to be observable which is needed for this scheme to be implementable - see $\S$ VIII).

More precisely, in a Poisson MANET, Opportunistic Aloha with random MAC threshold can be described by an independently marked P.p.p. $\widetilde{\Phi}=\left\{\left(X_{i}, \theta_{i}, y_{i}, \mathbf{F}_{i}\right)\right\}$, where $\left\{\left(X_{i}, y_{i}, \mathbf{F}_{i}\right)\right\}$ is as in (1)-(4) of Section II, with item (2) replaced by:

(2') The medium access indicator $e_{i}$ of node $i\left(e_{i}=1\right.$ if node $i$ is allowed to transmit and 0 otherwise) is the following function of the virtual power $F_{i}^{i}: e_{i}=$ $\mathbb{I}\left(F_{i}^{i}>\theta_{i}\right)$, where $\left\{\theta_{i}\right\}$ are new random i.i.d. marks of the point process of transmitters, independent of everything else. A special case of interest is that with $e_{i}=\mathbb{I}\left(F_{i}^{i}>\theta\right)$, where $\theta$ is some deterministic threshold.

We still assume that for each $i$, the components of $\left(F_{i}^{j}, j\right)$ are i.i.d. Note that $\left\{e_{i}\right\}$ are again i.i.d. marks of the point process $\widetilde{\Phi}$ (which of course depend on the marks $\left.\left\{\theta_{i}, F_{i}^{i}\right\}\right)$.

In what follows we will also assume that for each $i$ the coordinates of $\left(F_{i}^{j}, j\right)$ are i.i.d. (cf. assumption (4) of the plain Aloha model of Section II).

The set of transmitters is hence a Poisson p.p. $\Phi^{1}$ (different from that in Section II) with intensity $\lambda \mathbf{P}(F>$ $\theta$ ) (where $F$ is a typical $F_{i}^{i}$ and $\theta$ a typical $\theta_{i}$, with $(F, \theta)$ independent). Thus in order to compare Opportunistic Aloha to the plain Spatial Aloha described in Section II, one can take $p=\mathbf{P}\{F>\theta\}$, where $p$ is the MAP of plain Aloha, which guarantees the same density of (selected) transmitters at a given time slot. 


\section{B. Coverage - Outage}

Note that the virtual power emitted by any node to its receiver, given it is selected by Opportunistic Aloha (i.e. given $e_{i}=1$ ) has for law the distribution of $F$ conditional on $F>\theta$. Below, we will denote by $F_{\theta}$ a random variable with this law.

However, by independence of $\left(F_{i}^{j}, j\right)$, the virtual powers $F_{i}^{j}, j \neq i$, toward other receivers are still distributed as $F$. Consequently, the interference $I_{i}^{1}$ experienced at any receiver has exactly the same distribution as in plain Aloha. Hence, the probability for a typical transmitter to cover its receiver can be expressed by the following three independent generic random variables

$$
\widehat{p}_{c}\left(r, \lambda_{1}, T\right)=\mathbf{P}\left\{F_{\theta}>T l(r)\left(I^{1}+W\right)\right\},
$$

where $I^{1}$ is the generic shot-noise generated by Poisson p.p. with intensity $\lambda_{1}=\mathbf{P}\{F>\theta\} \lambda$ and (nonconditioned) fading variables $F_{j}$ (as in (2.4)).

We begin our analysis of Opportunistic Aloha by a comparison of $\widehat{p}_{c}\left(r, \lambda_{1}, T\right)$ and $p_{c}\left(r, \lambda_{1}, T\right)$ of plain Aloha when all parameters $(T, W, r$, etc.) are the same. To get more insight we assume first Rayleigh fading. In this case $F$ is exponential with parameter $\mu$ and since $\theta$ is independent of $F$, by the lack of memory property of the exponential variable, given that $F>\theta$ the variables $\theta$ and $F-\theta$ are independent. Moreover, the conditional distribution of $F-\theta$ given $F>\theta$ is also exponential with parameter $\mu$. Denote by $\widetilde{\theta}$ the conditional law of $\theta$ given that $F>\theta$. Consequently in the Rayleigh fading case (7.1) can be rewritten as

$$
\widehat{p}_{c}\left(r, \lambda_{1}, T\right)=\mathbf{E}\left[e^{-\mu\left(T l(r)\left(I^{1}+W\right)-\widetilde{\theta}\right)^{+}}\right],
$$

where $a^{+}=\max (a, 0)$. Comparing (7.2) to the middle expression in (2.5) it is clear that the opportunistic scheme does better than plain Aloha with MAP $p$ such that $p=\mathbf{P}(F>\theta)=E\left(e^{-\mu \theta}\right)=\mathcal{L}_{\theta}(\mu)$. Indeed, the intensity of transmitters is the same in both cases, and thus the laws of $I^{1}$ coincide in both formulas.

In order to evaluate how much better Opportunistic Aloha does in the Rayleigh case, we will particularly focus on the following two simple examples:

- that where $\theta$ is constant, where $\widetilde{\theta}=\theta$,

- that where $\theta$ is exponential with parameter $\nu$ where $\widetilde{\theta}$ is exponential of parameter $\mu+\nu$.

In the latter case we have:

Proposition 7.1: Assume the Poisson bipolar network model of Section II-A with Opportunistic Aloha MAC given by (2') above. Assume Rayleigh fading (exponential $F$ with parameter $\mu$ ) and exponential distribution of $\theta$ with parameter $\nu$. Then

$$
\begin{aligned}
\widehat{p}_{c}\left(r, \lambda_{1}, T\right) \\
=\frac{\mu+\nu}{\nu} \mathcal{L}_{I^{1}}(\mu T l(r)) \mathcal{L}_{W}(\mu T l(r)) \\
\quad-\frac{\mu}{\nu} \mathcal{L}_{I^{1}}((\mu+\nu) T l(r)) \mathcal{L}_{W}((\mu+\nu) T l(r)),
\end{aligned}
$$

where $\mathcal{L}_{I^{1}}$ is given by (2.6) with $\lambda_{1}=\lambda \nu /(\mu+\nu)$. If moreover $W \equiv 0$ and the path-loss (2.1) is assumed, then

$$
\begin{aligned}
\widehat{p}_{c}\left(r, \lambda_{1}, \nu\right)= & \frac{\mu+\nu}{\nu} \exp \left\{-\lambda_{1} T^{2 / \beta} r^{2} K(\beta)\right\} \\
& -\frac{\mu}{\nu} \exp \left\{-\lambda_{1}\left(\frac{(\mu+\nu) T}{\mu}\right)^{2 / \beta} r^{2} K(\beta)\right\},
\end{aligned}
$$

with $\lambda_{1}$ as above.

Proof: Note that $\Phi^{1}$ is a Poisson p.p. of intensity $\lambda \nu /(\nu+\mu)$ and $\widetilde{\theta}$ is exponential of parameter $\mu+\nu$. Using (7.2) we have hence

$$
\begin{array}{r}
\widehat{p}_{c}\left(r, \lambda_{1}, T\right) \\
=\mathbf{E}\left[\int_{0}^{T l(r)\left(I^{1}+W\right)}(\mu+\nu) e^{-(\mu+\nu) x} e^{-\mu\left(T l(r)\left(I^{1}+W\right)-x\right)} \mathrm{d} x\right] \\
+\mathbf{E}\left[\int_{T l(r)\left(I^{1}+W\right)}^{\infty}(\mu+\nu) e^{-(\mu+\nu) x} \mathrm{~d} x\right] \\
=\frac{\mu+\nu}{\nu} \mathbf{E}\left[e^{-\mu T l(r)\left(I_{1}+W\right)}-e^{-(\mu+\nu) T l(r)\left(I_{1}+W\right)}\right] \\
+\mathbf{E}\left[e^{-(\mu+\nu) T l(r)\left(I^{1}+W\right)}\right]
\end{array}
$$

which completes the proof.

Note that, as expected, when letting $\nu$ tend to infinity, under mild conditions, the first expression of the last proposition tends to $\mathcal{L}_{I^{1}}(\mu T l(r)) \mathcal{L}_{W}(\mu T l(r))$, namely the formula (2.7) of plain Aloha with $\lambda_{1}=\lambda$ or equivalently $p=1$.

For a general fading and distribution of $\theta$ (for instance deterministic) the following result can be proved along the same lines as Proposition 2.2.

Proposition 7.2: Assume the Poisson bipolar network model of Section II-A with Opportunistic Aloha MAC given by (2') above. Take the same assumptions as in Proposition 2.2 except that the condition on $F$ in the first item is replaced by the following one

- $\mathbf{E}\left(F_{\theta}\right)<\infty$ and $F_{\theta}$ admits a square integrable density.

Then

$$
\begin{aligned}
\widehat{p}_{c}\left(r, \lambda_{1}, T\right)=\int_{-\infty}^{\infty} \mathcal{L}_{I^{1}} & (2 i \pi l(r) T s) \mathcal{L}_{W}(2 i \pi l(r) T s) \\
& \times \frac{\mathcal{L}_{F_{\theta}}(-2 i \pi s)-1}{2 i \pi s} \mathrm{~d} s
\end{aligned}
$$

In the case of Rayleigh fading and deterministic $\theta$, the 


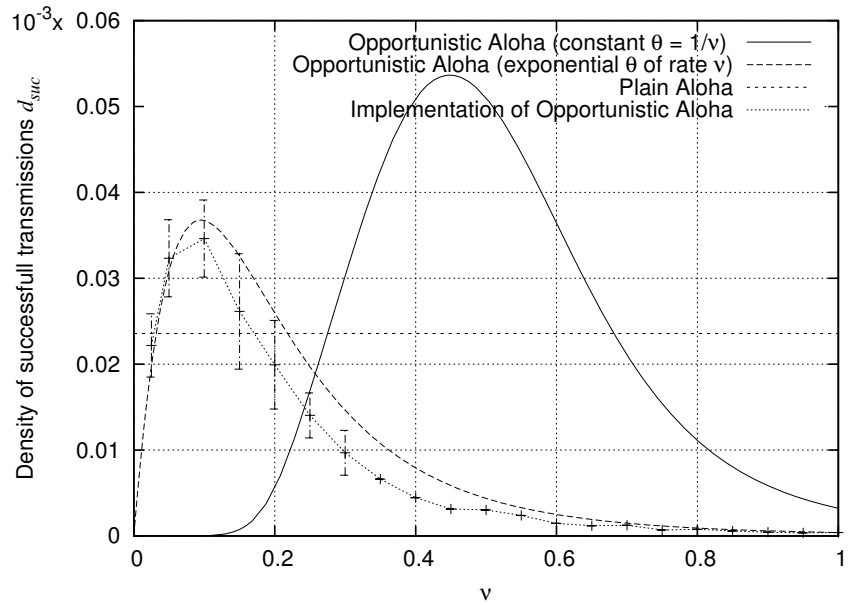

Fig. 2. The density of successful transmissions $d_{s u c}$ of Opportunistic Aloha for various choices of $\theta$. The propagation model is (2.1). We assume Rayleigh fading with mean 1 and $W=0, \lambda=0.001$, $T=10 \mathrm{~dB}, r=\sqrt{1 / \lambda}$ and $\beta=4$. For comparison the constant value $\lambda_{\max } p_{c}\left(r, \lambda_{\max }\right)$ of plain Aloha is plotted. The implementation of Opportunistic Aloha is described in Section VIII.

last theorem can be used since $F_{\theta}$ is then the convolution of a deterministic (equal to $\theta$ ) and an exponential (with parameter $\mu$ ) law, which satisfies the assumptions of the proposition. In this case $\mathcal{L}_{F_{\theta}}(s)=e^{-s \theta} \frac{\mu}{\mu+s}$. Obviously it can be also used when $\theta$ is exponentially distributed with intensity $\nu$. In particular for Rayleigh fading we have then $\mathcal{L}_{F_{\theta}}(s)=\frac{\mu+\nu}{\mu+\nu+s} \frac{\mu}{\mu+s}$.

Example 7.3: Assume Rayleigh fading. In Figure 2 we plot the density of successful transmissions $d_{s u c}$ in function of the parameter $\nu$ for three different scenarios:

1) Opportunistic Aloha with a deterministic threshold $\theta$ with value $1 / \nu$, where $d_{\text {suc }}=\lambda_{1} \widehat{p}_{c}\left(r, \lambda_{1}, \nu\right)$, with $\lambda_{1}=\lambda e^{-\frac{\mu}{\nu}}$ and $\widehat{p}_{c}\left(r, \lambda_{1}, \nu\right)$ given by Proposition 7.2 ;

2) Opportunistic Aloha with a random exponential threshold with parameter $\nu$, where $d_{\text {suc }}=$ $\lambda_{1} \widehat{p}_{c}\left(r, \lambda_{1}, \nu\right)$, with $\lambda_{1}=\frac{\lambda \nu}{\mu+\nu}$ and $\widehat{p}_{c}(r, \lambda, \nu)$ given by Proposition 7.1;

3) Plain Aloha where $d_{\text {suc }}=1 /\left(e K(\beta) r^{2} T^{2 / \beta}\right)$ is the optimal density of successful transmissions as obtained in Corollary 4.1 (this is of course a constant in $\nu$ ).

In the particular case considered in this figure, the density of transmitters covering their target receiver is approx. 56\% larger in the optimal opportunistic scheme with exponential threshold than in plain Aloha and 134\% larger in the deterministic case.

It may look surprising that the curves for the random exponential and the deterministic threshold cases (1 and 2 above) differ so much. One should bear in mind the fact that the two associated MAPs are quite different:

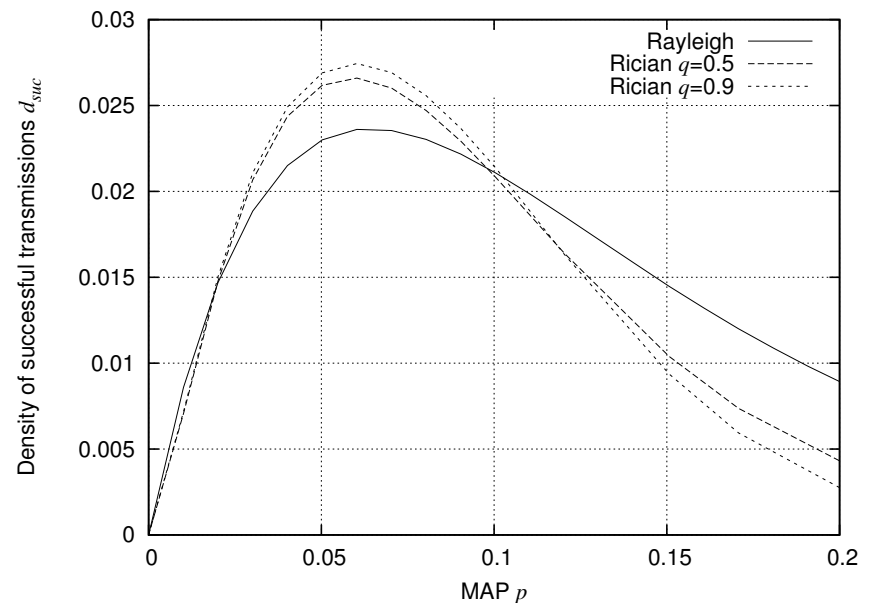

Fig. 3. Density of successful transmissions $d_{\text {suc }}$ for plain Aloha in function of $p$ in the Rayleigh and the Rician (with $q=1 / 2$ and $q=.9$ ) fading cases; $\lambda=r=1, W-0$, and $T=10 \mathrm{~dB}, \beta=4$.

$p=\frac{\nu}{\mu+\nu}$ in the former case and $p=e^{-\frac{\mu}{\nu}}$ in the latter.

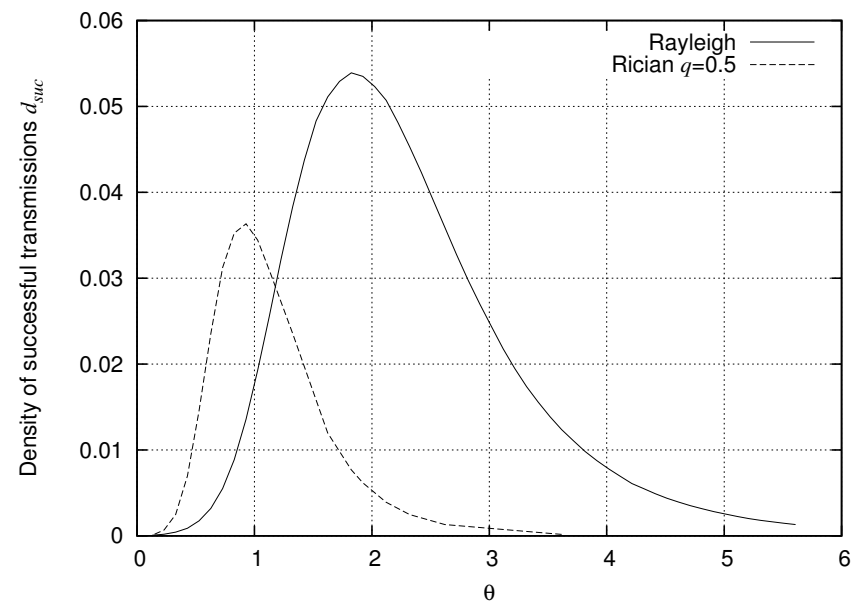

Fig. 4. Density of successful transmissions $d_{\text {suc }}$ for Opportunistic Aloha in function of the deterministic threshold $\theta$ in the Rayleigh and Rician (with $q=1 / 2$ ) fading cases; other parameters as on Figure 3.

\section{Example 7.4 (Rayleigh versus Rician fading):}

Figure 3 compares the density of success for Rayleigh and Rician fading in the plain Aloha case. For this, we use the representations of Proposition 2.1 and Proposition 2.2 respectively. In the Rayleigh case, $F$ is exponential with mean 1 . In the Rician case $F=q+(1-q) F^{\prime}$, with $0 \leq q \leq 1$, where $F^{\prime}$ is exponential with mean 1 and $q$ represents the part of the energy received on the line-of-sight. The density of success is plotted in function of $p$. We again observe that higher variances are beneficial for high densities of transmitters (which is here equivalent to the far field case) and detrimental for low densities. However here, in each case, there is an optimal MAP, and when 
properly optimized, Spatial Aloha does better for lower variances (i.e. for Rician fading with higher $q$ ).

Figure 4 compares the density of success of Opportunistic Aloha for Rayleigh and Rician $(q=.5)$ fading. (The Rician case with $q=.9$ has thresholds $\theta$ larger than .9 and leads to very small densities of success; it is not displayed.) The two curves are based on Proposition 7.2. Note first that for the two considered cases the density of transmitters are quite different: $(\exp (-\theta)$ in the Rayleigh case and $\exp (-2 \theta)$ in the Rician case, for $\theta>1 / 2$ ), which explain why the shapes of the curves are so different. Here, we see the opposite phenomenon compared to what was observed above: when properly optimized, Opportunistic Aloha does better when the fading variance increases, namely does much better for Rayleigh fading than for Rician fading with $q=0.5$. This is in fact quite natural since the aim of Opportunistic Aloha is to leverage diversity: more fading diversity/variance is hence beneficial to this protocol when properly tuned.

\section{Throughput}

The following result on the throughput of Opportunistic Aloha is a corollary of Proposition 7.2 and formula (2.12).

Corollary 7.5: Under assumptions of Proposition 7.2, the mean throughput can be expressed as:

$$
\begin{aligned}
\tau= & \int_{0}^{\infty} \int_{-\infty}^{\infty} \mathcal{L}_{I^{1}}\left(2 i \pi l(r)\left(e^{t}-1\right) s\right) \\
& \times \mathcal{L}_{W}\left(2 i \pi l(r)\left(e^{t}-1\right) s\right) \frac{\mathcal{L}_{F_{\theta}}(-2 i \pi s)-1}{2 i \pi s} \mathrm{~d} s \mathrm{~d} t .
\end{aligned}
$$

Since the assumptions of the last result hold in both deterministic and exponential $\theta$ case given Rayleigh fading, we can use (7.4) to evaluate the density of throughput in both cases.

Example 7.6: Figure 5 plots the density of throughput $d_{\text {throu }}$ for Rayleigh fading and the same three cases of $\theta$ as in Example 7.3. In the particular case considered in this figure, the density of throughput is approx. $48 \%$ larger in the optimal opportunistic scheme with exponential threshold than in plain Aloha and 93\% larger in the deterministic case.

Remark: The deterministic threshold case seems to always outperform the exponential threshold case when both are tuned optimally.

\section{A Possible Implementation OF OPPORTUNISTIC ALOHA}

The stochastic geometry estimates of the last section determine the performance of an ideal implementation

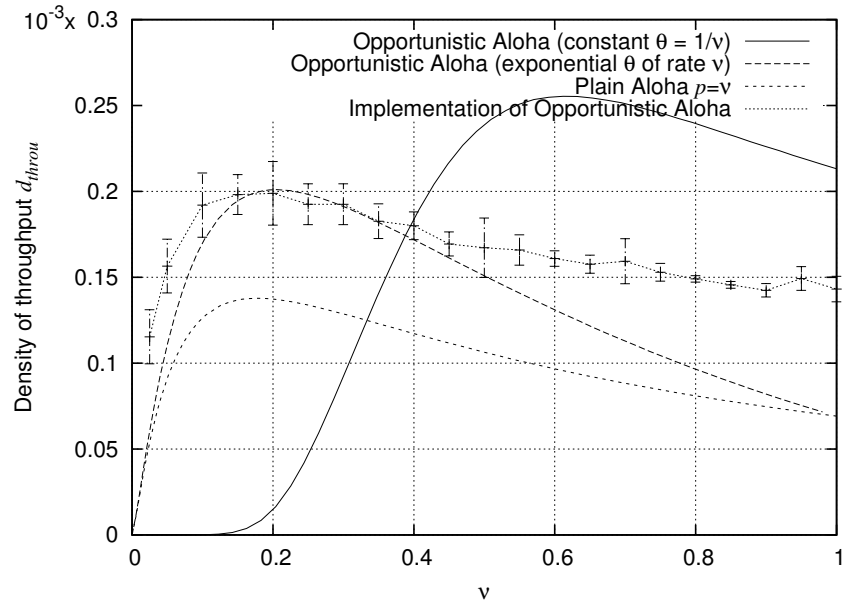

Fig. 5. The density of throughput of Opportunistic Aloha as a function of its parameter $\nu$ and that of plain Aloha. Assumptions are as on Figure 2. The implementation of Opportunistic Aloha is described in Section VIII.

of Opportunistic Aloha. The main question is of course whether the quality of the channel can be evaluated efficiently enough within the MANET setting. The aim of the present section is to outline a scheme allowing one to realize this estimation.

\section{A. Assumptions on Fading}

We make the following assumptions:

- Fading conditions change very often and more precisely these conditions may change from a time slot to the next; thus in order to implement the protocol, we need a scheme to estimate the fading between a sender and its receiver in each time slot.

- Fading is symmetrical i.e. the fading from transmitter to receiver is the same as the one from receiver to transmitter.

\section{B. Scheme for the Selection of Good Channels}

In order to identify sender-receiver pairs with good channel conditions under the assumption of fast fading changes, we propose to use a Request-To-Send (RTS) Clear-To-Send (CTS)-like approach. More precisely the Aloha slot is divided into three parts. The sender uses the first part of the slot to send an RTS packet containing the identity of the sender-receiver pair. As we will see below, the scheme is such that the receiver correctly receives the RTS packet if the channel conditions are good enough; upon reception of the RTS packet, the receiver uses the second part of the slot to send a CTS packet back to the sender to inform the latter of the fact that good conditions are observed. The sender is selected for transmission if both RTS and CTS were received successfully. The third 


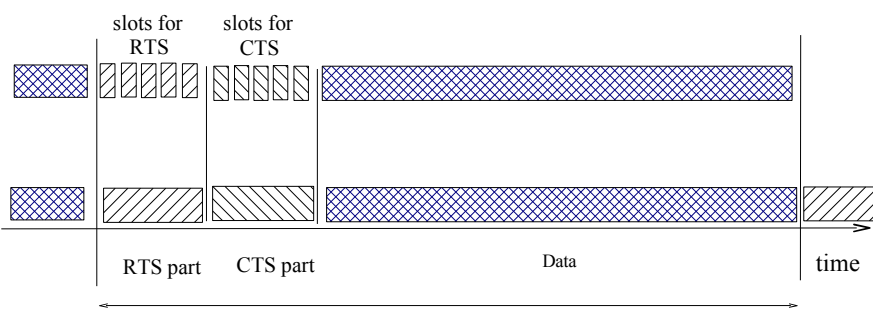

Aloha slot

Fig. 6. Structure of the time slot.

and final part of the slot is used by the sender to transmit the payload if selected.

This division of the Aloha slot is summarized in Figure 6.

The correct reception of the RTS packet by the receiver will not be judged using the conventional SINR criterion which is usually employed to validate the proper reception of a packet. We will rather measure the total power received by this node. That is the sum of the power of the signal transmitted by the sender and of that of the interference generated by all the other nodes. If this total power is larger than a pre-determined threshold, called the RTS threshold and denoted by $T_{R T S}$, the RTS packet is assumed to be correctly received. The rationale for this is the following: if the power of interference is small, then a high power is equivalent to a high signal power and hence good fading conditions; more precisely, if there is no interference at all, the RTS threshold should be taken equal to $T_{R T S}=\theta / l(r)$ when one seeks for channels with fading larger than $\theta$ (see $\S$ VII).

In such a case, the receiver sends a CTS packet in the second part of the Aloha slot, so as to inform the sender of the fact that it observes a good channel. Again, the proper reception of the CTS packet by the sender is not evaluated on the basis of SINR but rather on the basis of a received power larger than a pre-determined threshold called the CTS threshold and denoted by $T_{C T S}$. If interference is small, the symmetry assumption implies that one should also take $T_{C T S}=T_{R T S}=\theta / l(r)$.

Of course, as described above, the scheme only works if the interference is of low power both at the sender and at the receiver. In order to fulfil this requirement, we propose using a combination of CDMA codes and/or TDMA:

- CDMA: the RTS packet is sent using a random CDMA code chosen from a given set of CDMA codes. The reception of the RTS packet employs parallel matched filters using all the CDMA codes in the given set of codes. The reception of the RTS and CTS packets is then decided based on the value of the power received in the matched filter of the CDMA code on which the RTS packet was encoded. The use of the CDMA codes leads to a reduction of the interference power by a factor directly linked to the coding gain of the CDMA codes. CDMA codes of reasonable length can easily provide gains from 5 to 100 .

- TDMA: another way to decrease the impact of interference is to use a Time Division Multiple Access technique. These packets are sent in mini slots as shown on the top of Figure 6. The sender randomly selects a mini slot to send its RTS packet. The receiver follows the signal strength during the whole RTS part of the Aloha slot. The reception of the RTS packet is decided based on the total power during the RTS mini slot on which this RTS packet was transmitted. A comparison of this maximum total power with the RTS threshold decides whether the RTS has been properly received. A similar technique is used for CTS packets based on appropriate mini slots. The gain obtained by this technique is equal to the number of mini-slots created in the RTS (or CTS) part.

- CDMA and TDMA: it is possible to mix the use of TDMA access for RTS/CTS packets with the use of CDMA codes. In this case the gain against the interference is the product of the gain obtained with the CDMA codes and of the gain obtained the TDMA approach for ther RTS/CTS packets.

Note that the RTS/CTS messages proposed above are not supposed to create any exclusion zones (like in CSMA) but to discover the quality of the channel between the emitter and its potential receivers. In fact, CDMA and TDMA techniques are proposed to separate neighboring selection processes.

\section{Discrete Event Simulation}

The simulated scenario is the following:

- Path-loss: (2.1) with $A=1, \beta=4$;

- Thermal noise: $W \equiv 0$;

- Threshold: $T=10 \mathrm{~dB}$;

- Fading: Rayleigh with $\mu=1$;

- RTS-CTS: we set to $N=50$ the gain against the interference that can be obtained with the combination of CDMA codes and TDMA. In the simulation results, we apply a correction corresponding to an overhead of $15 \%$ to take the time lost in the RTS and CTS parts of the slot into account. We use the same threshold to qualify the reception of the RTS and the CTS packets, $T_{R T S}=T_{C T S}=\theta / l(r)$. In 


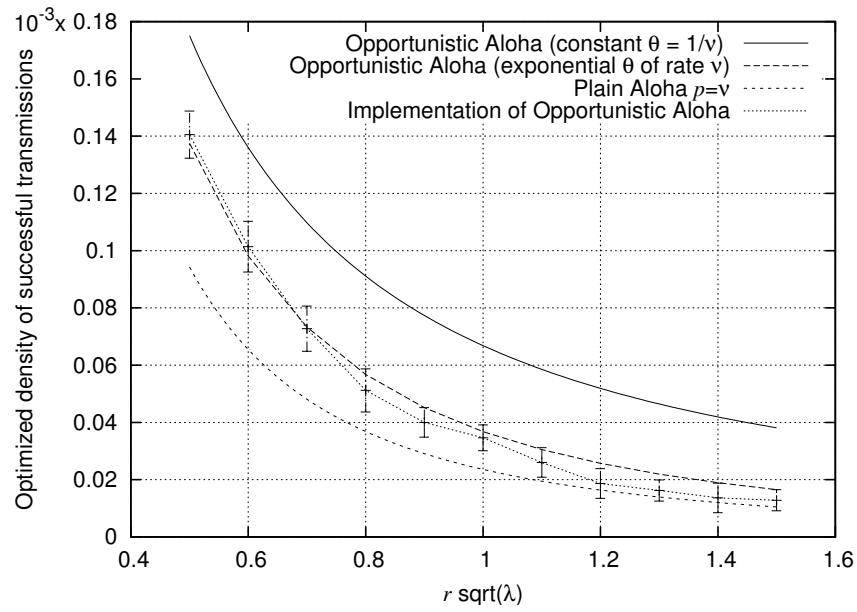

Fig. 7. Optimized density of successful transmissions for plain Aloha and Opportunistic Aloha versus $r$ in fraction of $1 / \sqrt{\lambda}$. Other assumptions as on Figure 2.

the simulations, $\theta$ is either constant $1 / \nu$ or randomly selected according to an exponential law of rate $\nu$.

- Point pattern: Poisson point process of intensity $\lambda=0.001$ on a square $[0,1000] \times[0,1000]$.

We study the transmission from a given node locate at the center of the square to a receiver located at a distance $r$ meters from the transmitter, as done in the analytical model.

In Figure 2, we plot the density of successful transmissions obtained by the implementation of Opportunistic Aloha in the case with random exponential threshold (this is the curve with confidence interval). We observe that the implementation of Opportunistic Aloha significantly outperforms plain Aloha when $\nu$ is correctly chosen. This implementation of Opportunistic Aloha remains less efficient than the theoretical model. We also notice that the implementation of Opportunistic Aloha exhibits the same kind of behavior as the theoretical model.

In Figure 5, we evaluate the density of throughput of the implementation of Opportunistic Aloha. We have the same observations as for Figure 2. Once again the implementation of Opportunistic Aloha very significantly outperforms plain Aloha .

In Figure 7 and Figure 8, we study the density of successful transmissions and the density of throughput for different values of $r$. In these figures we evaluate the performance of plain Aloha (with $p=1 / \nu$ ), of the theoretical model of Opportunistic Aloha $(\theta=1 / \nu$ or $\theta$ exponentially distributed of rate $\nu$ ) and of the implementation of Opportunistic Aloha (for which we only plot the exponential threshold case). For all values of $r$, the theoretical models are optimized by selecting

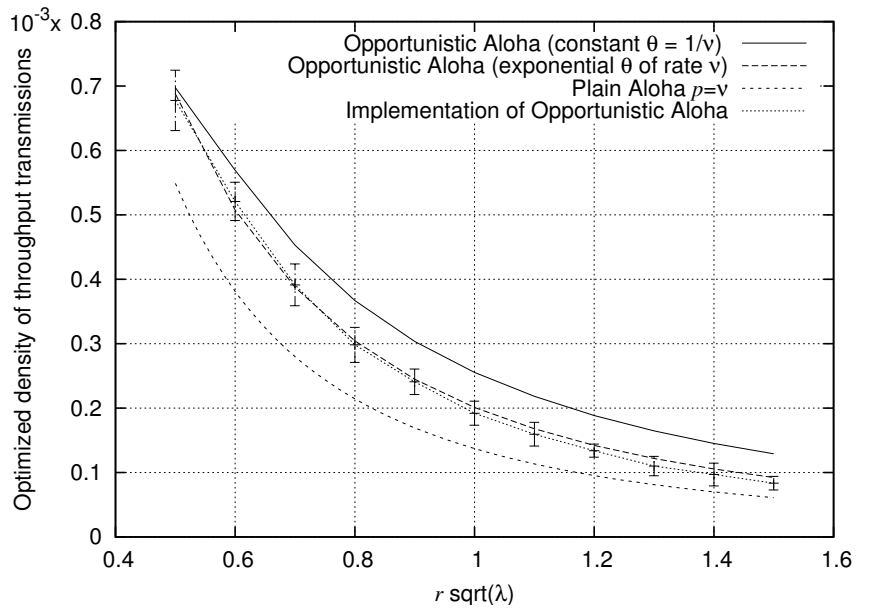

Fig. 8. Optimized density of throughput for Aloha and Opportunistic Aloha versus $r$ in fraction of $1 / \sqrt{\lambda}$. Other assumptions as on Figure 2.

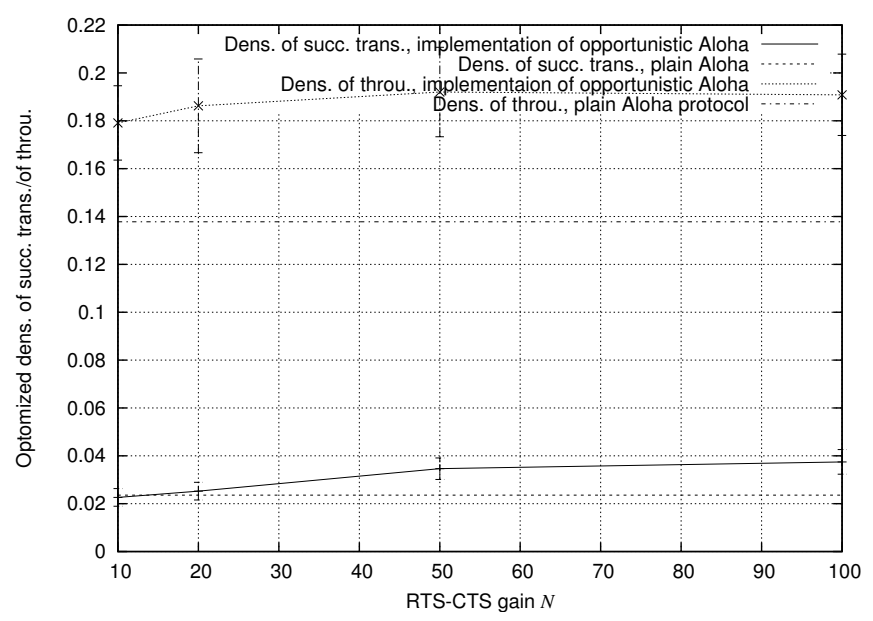

Fig. 9. Density of successful transmissions and density of throughput for the implementation of Opportunistic Aloha in function of the RTS-CTS gain $N$ against the interference. Theoretical values for plain Aloha model are given for comparison.

the best value of $\nu$ (as given in $\S \mathrm{IV}$ and $\S \mathrm{V}$ for plain Aloha and as obtained from the formulas of $\S \mathrm{VII}$ for Opportunistic Aloha). For the implementation of Opportunistic Aloha a rough optimization at the best value of $\nu$ is performed.

We observe that Opportunistic Aloha outperforms plain Aloha for all transmission radii $r$. We also note that the implementation of Opportunistic Aloha tends to offer results close to those of the theoretical model.

In Figure 9, we study the effect of the gain against interference $N$. For these results, the implementation of Opportunistic Aloha is roughly optimized w.r.t. $\nu$. We observe the following:

- For $N \geq 20$, the proposed implementation of Opportunistic Aloha improves significantly on plain 
Aloha: the improvement is appr. from $10 \%$ to $50 \%$ for the density of successful transmissions and of $35 \%$ for the density of throughput.

- For $10 \leq N \leq 20$, the implementation of Opportunistic Aloha improves the density of throughput significantly and offers a marginal improvement in the density of successful transmissions.

- The implementation of Opportunistic Aloha based on a fixed value of $\theta$ allows one to obtain an improvement of $10 \%$ in the density of successful transmission even for $N=10$.

Remark: Notice that the RTS-CTS phase can be used to select the best receiving antenna at the receiver if it has more than one receiving antenna. This may improve the performance of Opportunistic Aloha and increase the gain of this scheme over plain Aloha.

\section{CONCLuding Remarks}

\section{A. General Remarks}

The aim of this subsection is to discuss how to relax some of the simplifying assumptions adopted in the present paper and more generally how to extend our approach to more realistic scenarios or to further metrics.

a) Extension to non slotted systems: We assumed time to be slotted. Time-slots are required in e.g. TDMA (Time Division Multiple Access) systems and one of the well known advantages of Aloha is that it does not require slotted time. In order to model non-slotted Aloha, one has to take into account the fact that interference (and thus SINR) can vary during a given transmission as some other transmissions may start or terminate. A more detailed packet reception model is hence needed. For example, if one assumes a coding with some sufficient interleaving, then one can consider that it is the averaged SINR, where the averaging is over the whole packet reception period, that determines the success of reception. In this case a mathematical analysis of nonslotted Aloha is possible e.g. along the lines presented in [12], [13].

b) Extensions of the bipolar model and to multihop scenario: Our bipolar model is meaningful for a multi-hop network in the sense that it allows one to analyse the performance of such a network "at some arbitrary time slot". The transmitters considered in this slot are relay nodes and not necessarily the sources of the transmitted packets. Similarly, the receivers need not be the final destinations. The fact that all receivers are at the same distance from their emitters presents some simplification that can easily be relaxed by considering random (independent) distances. This one-hop analysis can be used to deduce the performance of a routing in a multi-hop network under the assumption of high node mobility; in this case, the configurations of nodes in two different slots might be taken as independent and Poisson and one can then use independence to analyze the fate of a packet along its route, under some simplified routing models. If this high mobility assumption cannot be made, multi-hop analysis complicates considerably (see e.g. the cross-layer model of Aloha MAC with opportunistic routing in the second part of [1] and in [14]). We decided not to address these routing issues in the present paper.

c) Extension to models with external noise: Some of our mathematical results (e.g. these concerning scaling laws) require no external noise $W=0$. Our numerical examples are also given under this assumption. This is not however essential for the paper. Most of our quantitative analysis allows for an arbitrary distribution of $W$. Moreover, our numerical results (not presented in this paper) show that as long as $W$ represents only the thermal noise (i.e. is of order of $10^{-10} \mathrm{~mW}$ or smaller), it can be neglected.

d) Refined averaging: Stochastic geometry tools were used to evaluate various averages (over time and space). However, these tools can also be used to provide some qualitative analysis of any given "random" configuration of nodes. For example, one can show that if the fading variable $(F)$ has unbounded support or if the external noise is negligible $(W=0)$, then Aloha provides some positive probability of successful transmission (and thus throughput) on an arbitrary distance $r$ for each user in the Poisson configuration. In such a statement, the averaging is over the MAC status of the other users $\left(e_{i}\right)$ and not over their locations. For more on the matter, see the IEEE IT version of $[1, \S \mathrm{VII}]$.

\section{B. Conclusions}

Stochastic geometry provides a versatile framework for the performance analysis of MAC protocols for large MANETs. This was exemplified in the present paper where we both provided several new analytical results on Spatial Aloha and outlined a new variant of the protocol leveraging a real time estimation of the potential channels to be scheduled at any given time.

Among the key advantages of the developed machinery, we would like to stress that it allows one to optimize and to compare protocols in a purely analytic and hence very synthetic way: in the same way as queueing theory can be used to compare time averages of e.g. a blind and an adaptive scheduling scheme in a queue, the spatial averages computed by stochastic geometry allow one to evaluate how much better an adaptive version of a MAC protocol (Opportunistic Aloha) works compared to the 
plain version (Spatial Aloha). This type of analysis leads to the identification of important qualitative phenomena, like the very beneficial role of fading variability in opportunistic schemes, which seem to be new within this context.

Among the potential research directions, we would like to quote the stochastic geometry analysis of adaptive variants of CSMA/CA schemes, in continuation of the analysis based on Matern point processes proposed in [15].

\section{REFERENCES}

[1] F. Baccelli, B. Blaszczyszyn, and P. Mühlethaler, "An Aloha Protocol for Multihop Mobile Wireless Networks," in Proceedings of the Allerton Conference, University of Illinois, Urbana Champaign, November 2003, and IEEE Transactions on Information Theory, 52(2):421-436, 2006.

[2] N. Ehsan and R. L. Cruz, "On the optimal SINR in random access networks with spatial reuse," in Proceedings of the CISS 2006 Conference, Princeton University, NJ, USA, 2006.

[3] P. Jacquet, "Realistic wireless network model with explicit capacity evaluation,” http://hal.inria.fr/inria-00203339, INRIA, Rapport de recherche RR-6407, 2008.

[4] H. Koskinen, T. Tirronen, and J. Virtamo, "Exact distribution of Poisson shot noise with constant marks under powr-law attenuation," IEEE Electronics Letters, vol. 42, 2006.

[5] A. Hunter, J. Andrews, and S. Weber, "Capacity scaling of ad hoc networks with spatial diversity," submitted for publication, 2008.

[6] D. Tse and P. Viswanath, Foundamentals of Wireless Communication. Cambridge University Press, 2005.

[7] D. J. Daley and D. Vere-Jones, An Introduction to the Theory of Point Processes, vol. 1. Springer, 2003.

[8] D. Stoyan, W. Kendall, and J. Mecke, Stochastic Geometry and its Applications. Chichester: Wiley, 1995.

[9] F. Baccelli and B. Blaszczyszyn, "On a coverage process ranging from the Boolean model to the Poisson-Voronoi tessellation, with applications to wireless communications," $A d v$. Appl. Prob., vol. 33, pp. 293-323, 2001.

[10] W. Feller, An Introduction to Probability Theory and its Applications, volume II. Wiley, 1971.

[11] P. Brémaud, Mathematical Principles of Signal Processing. New York: Springer, 2002.

[12] B. Błaszczyszyn and B. Radunović, "M/D/1/1 loss system with interference and applications to transmit-only sensor networks," in Proc. of SpaSWiN, 2007.

[13] _ , "Using transmit-only sensors to reduce deployment cost of wireless sensor networks," in Proc. of IEEE INFOCOM, Phoenix, AZ, 2008.

[14] F. Baccelli, B. Błaszczyszyn, and P. Mühlethaler, "On the performance of time-space opportunistic routing in multihop mobile ad hoc networks," in Proc. of IEEE WiOpt, Berlin, April 2008.

[15] H. Nguyen, F. Baccelli, and D. Kofman, "A stochastic geometry analysis of dense ieee 802.11 networks," in Proceedings of IEEE Infocom 2007, 2007, pp. 1199-1207.
François Baccelli's research interests are in the theory of discrete event dynamical networks and in the modeling and performance evaluation of computer and communication systems. He coauthored more than 100 publications in major international journals and conferences, as well as a 1994 Springer Verlag book on queueing theory, jointly with P. Bremaud, the second edition of which appeared in 2003, and a 1992 Wiley book on the max plus approach to discrete event networks, with G. Cohen, G.J. Olsder and J.P. Quadrat.

He was the head of the Mistral Performance Evaluation research group of INRIA Sophia Antipolis, France, from its creation to 1999. He was/is a partner in several European projects including IMSE (Esprit 2), ALAPEDES (TMR) and EURONGI (Network of Excellence), and was the coordinator of the BRA Qmips European project.

He is currently INRIA Directeur de Recherche in the Computer Science Department of Ecole Normale Supérieure in Paris, where he started the TREC group (théorie des reśeaux et communications) in 1999.

His current research interest are focused on the analysis of large wireless networks and the design of scalable and reliable application layers based on the current point to point transport mechanisms, and on the development of new tools for the modeling of protocols with spatial components in wireless networks such as coverage and power control in CDMA and MAC protocols in mobile had hoc networks.

F. Baccelli was awarded the 2002 France Telecom Prize by the French Academy of Sciences.

E-mail: Francois.Baccelli@ens.fr

WWW: www.di.ens.fr/ ־ baccelli

Bartłomiej Błaszczyszyn received his MS and Ph.D degree in applied mathematics from University of Wrocław (Pologne) in 1990 and 1995, respectively. He is an Assistant Professor in Mathematical Institute University of Wrocław and a staff member of joint INRIAENS research group in Paris. His professional interests are in applied probability and in particular in modeling of communication networks by means of stochastic geometry.

E-mail: Bartek.Blaszczyszn@ens.fr

WWW: www.di.ens.fr/ ` blaszczy

Paul Mühlethaler was born in February 1961. He graduated from the Ecole Polytechnique in 1984. He received his PhD in 1989 from the University Paris Dauphine and his research director qualification in 1998. His research topics mainly concern protocols for wireless networks. He has actively worked at ETSI and IETF for the HiPERLAN and OLSR standards. He is now active in the European standardization for vehicular networks. Another important aspect of his activity concerns models and performance evaluations. He is particularly interested in gaining a greater insight into the achievable performances of multihop ad hoc networks and tracking all the possible optimizations of such networks.

E-mail: Paul.Muhlethaler@inria.fr

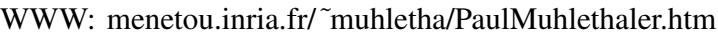




\section{LIST OF FIGURES}

1 A snapshot of bipolar MANET with Aloha MAC. ............... 4

2 The density of successful transmissions; Opportunistic vs plain Aloha. . . . . . . . 13

3 The density of successful transmissions in Aloha; Rayleigh vs Rician fading. . . . . . 13

4 The density of successful transmissions in Opportunistic Aloha; Rayleigh vs Rician fading. . . . . . . . . . . . 13

5 The density of throughput of Opportunistic Aloha in function of the channel threshold. 14

6 Structure of the time slot. . . . . . . . . 15

7 Optimized density of successful transmissions for Aloha and Opportunistic Aloha in function of the receiver distance. . . . . . 16

8 Optimized density of throughput for Aloha and Opportunistic Aloha in function of the distance. . . . . . . . . . . . 16

9 Impact of the RTS-CTS gain implemented in Opportunistic Aloha. . . . . . . . . . . . 16 